\title{
ORDINARY VARIETIES AND THE COMPARISON BETWEEN MULTIPLIER IDEALS AND TEST IDEALS
}

\author{
MIRCEA MUSTAŢ $\breve{A}$ AND VASUDEVAN SRINIVAS
}

\begin{abstract}
We consider the following conjecture: if $X$ is a smooth and irreducible $n$-dimensional projective variety over a field $k$ of characteristic zero, then there is a dense set of reductions $X_{s}$ to positive characteristic such that the action of the Frobenius morphism on $H^{n}\left(X_{s}, \mathcal{O}_{X_{s}}\right)$ is bijective. There is another conjecture relating certain invariants of singularities in characteristic zero (the multiplier ideals) with invariants in positive characteristic (the test ideals). We prove that the former conjecture implies the latter one in the case of ambient nonsingular varieties.
\end{abstract}

\section{$\S 1$. Introduction}

It has been known for about thirty years that there are close connections between classes of singularities that appear in birational geometry and such classes that appear in commutative algebra and, more precisely, in tight closure theory. Recall that in birational geometry, singularities are typically described in terms of a suitable resolution of singularities. On the other hand, tight closure theory describes the singularities in positive characteristic in terms of the action of the Frobenius morphism. The connection between the two points of view is very rich but still remains somewhat mysterious.

The best-known example of such a connection concerns rational singularities: it says that a variety has rational singularities if and only if it has $F$-rational type. ( $F$-rationality is a notion defined in positive characteristic via the tight closure of parameter ideals.) More precisely, suppose that $X$ is defined over a field $k$ of characteristic zero, and consider a model of $X$ defined over an algebra $A$ of finite type over $\mathbf{Z}$. For every closed point

Received January 16, 2011. Revised June 2, 2011. Accepted June 5, 2011.

2010 Mathematics Subject Classification. Primary 13A35; Secondary 14F18, 14 F30.

Mustaţă was partially supported by National Science Foundation grant DMS-0758454 and by a Packard Fellowship. Srinivas was partially supported by a J.C. Bose Fellowship of the Department of Science and Technology, India. 
$s \in \operatorname{Spec} A$, consider the corresponding reduction $X_{s}$ to positive characteristic. Then $X$ has rational singularities if and only if there is an open subset $U$ of Spec $A$ such that $X_{s}$ has $F$-rational singularities for every closed point $s \in U$. (The "if" part was proved in [Smi], while the "only if" part was proved independently in [Ha] and [MS].)

Other classes of singularities behave in the same fashion (see $[\mathrm{HW}$ ] for the comparison between Kawamata log terminal and strongly $F$-regular singularities). On the other hand, a more subtle phenomenon relates, for example, $\log$ canonical and $F$-pure singularities. It is known that if there is a (Zariski) dense set of closed points $S \subset \operatorname{Spec} A$ such that $X_{s}$ has $F$-pure singularities for all $s \in S$, then $X$ has $\log$ canonical singularities (see [HW]). The converse, however, is widely open, and in general the set of closed points $s \in \operatorname{Spec} A$ for which $X_{s}$ has $F$-pure singularities does not contain an open subset, even when it is dense. Furthermore, examples have made it clear that some subtle arithmetic phenomena are involved.

The main goal of our paper is to consider an arithmetic-geometric conjecture and to show that it implies a similar such connection, between multiplier ideals (invariants in characteristic zero) and test ideals (invariants in characteristic $p$ ). We believe that this gives a new perspective to the correspondence between the two sets of invariants, and we hope it points to a possible way of proving this correspondence.

Conjecture 1.1. Let $X$ be a smooth, connected $n$-dimensional projective variety over an algebraically closed field $k$ of characteristic zero. Given a model of $X$ over a $\mathbf{Z}$-algebra of finite type $A$, contained in $k$, there is a dense set of closed points $S \subseteq \operatorname{Spec} A$ such that the action induced by Frobenius on $H^{n}\left(X_{s}, \mathcal{O}_{X_{s}}\right)$ is bijective for every $s \in S$.

As we will show, in the above conjecture it is enough to consider the case $k=\overline{\mathbf{Q}}$ (see Proposition 5.3). We mention that it is expected that under the assumptions in the conjecture, there is a dense set of closed points $S \subseteq$ Spec $A$ such that for every $s \in S$, the smooth projective variety $X_{s}$ over $k(s)$ is ordinary in the sense of $[\mathrm{BK}]$. One can show that this condition implies that the action induced by Frobenius on each cohomology group $H^{i}\left(X_{s}, \mathcal{O}_{X_{s}}\right)$ is bijective. On the other hand, we hope that the property in Conjecture 1.1 will be easier to prove than the stronger property of being ordinary.

Before stating the consequence of Conjecture 1.1 to the relation between multiplier ideals and test ideals, let us recall the definitions of these ideals. 
Since our main result deals only with nonsingular ambient varieties, we review these concepts in this special case. Let $Y$ be a nonsingular, connected variety defined over an algebraically closed field of characteristic zero, and suppose that $\mathfrak{a}$ is a nonzero ideal on $Y$. Recall that a $\log$ resolution of $(Y, \mathfrak{a})$ is a projective birational morphism $\pi: X \rightarrow Y$, with $X$ nonsingular and $\mathfrak{a} \cdot \mathcal{O}_{X}=\mathcal{O}_{X}(-G)$, with $G$ a divisor, such that there is a simple normal crossings divisor $E$ on $X$, with both $G$ and $K_{X / Y}$ supported on $E$. Here $K_{X / Y}$ is the relative canonical divisor. Such resolutions exist by Hironaka's theorem, since $Y$ lives in characteristic zero. The multiplier ideal of $\mathfrak{a}$ of exponent $\lambda \geq 0$ is the ideal

$$
\mathcal{J}\left(Y, \mathfrak{a}^{\lambda}\right):=\pi_{*} \mathcal{O}_{X}\left(K_{X / Y}-\lfloor\lambda G\rfloor\right),
$$

where for any $\mathbf{R}$-divisor $E$, we denote by $\lfloor E\rfloor$ its round-down. It is a general fact that the definition is independent of the given resolution. These ideals have recently found many striking applications in birational geometry, mostly due to their connection with vanishing theorems (see [Laz]).

In positive characteristic, Hara and Yoshida [HY] introduced the notion of the (generalized) test ideal, relying on a generalization of the theory of tight closure. In this paper we use an equivalent definition due to Schwede [Sch]. This definition is particularly transparent in the case of an ambient nonsingular variety, when it is an immediate consequence of the description in $[\mathrm{BMS}]$.

Suppose that $Y$ is a nonsingular, connected variety over a perfect field $L$ of characteristic $p>0$, and suppose that $\mathfrak{a}$ is an ideal on $Y$. The Cartier isomorphism induces a surjective $\mathcal{O}_{X}$-linear map $t_{Y}: F_{*} \omega_{Y} \rightarrow \omega_{Y}$, where $F$ is the absolute Frobenius morphism. Iterating this $e$ times gives $t_{Y}^{e}: F_{*}^{e} \omega_{Y} \rightarrow$ $\omega_{Y}$. For any ideal $\mathfrak{b}$ on $Y$, and for every $e \geq 1$, the ideal $\mathfrak{b}^{\left[1 / p^{e}\right]}$ is defined by $t_{Y}^{e}\left(F_{*}^{e}\left(\mathfrak{b} \cdot \omega_{Y}\right)\right)=\mathfrak{b}^{\left[1 / p^{e}\right]} \cdot \omega_{Y}$. Given any $\lambda \geq 0$, it is easy to see that the sequence of ideals $\left(\left(\mathfrak{a}^{\left\lceil\lambda p^{e}\right\rceil}\right)^{\left[1 / p^{e}\right]}\right)_{e \geq 1}$ is nondecreasing, and therefore it stabilizes by the Noetherian property. The limit is the test ideal $\tau\left(Y, \mathfrak{a}^{\lambda}\right)$. (For a discussion of various analogies between test ideals, and multiplier ideals, see $[\mathrm{HY}]$.) The following is the main conjecture relating multiplier ideals and test ideals.

CONJECTURE 1.2. Let $Y$ be a nonsingular, connected variety over an algebraically closed field $k$ of characteristic zero, and let $\mathfrak{a}$ be a nonzero ideal on $Y$. Given a model for $Y$ and $\mathfrak{a}$ defined over a $\mathbf{Z}$-algebra of finite type $A$, contained in $k$, there is a dense set of closed points $S \subset \operatorname{Spec} A$ 
such that

$$
\tau\left(Y_{s}, \mathfrak{a}_{s}^{\lambda}\right)=\mathcal{J}\left(Y, \mathfrak{a}^{\lambda}\right)_{s}
$$

for all $s \in S$ and all $\lambda \geq 0$. Furthermore, if we have finitely many pairs as above $\left(Y^{(i)}, \mathfrak{a}^{(i)}\right)$, and corresponding models over Spec $A$, then there is a dense open subset of closed points in $\operatorname{Spec} A$ such that (1) holds for each of these pairs.

Two things are known. First, under the assumptions in the conjecture, there is an open subset of closed points in $\operatorname{Spec} A$ for which the inclusion $\subseteq$ in (1) holds for all $\lambda$. This was proved in $[\mathrm{HY}]$ and is quite elementary. (We give a variant of the argument in Section 3, using the equivalent definition in [Sch].) A second, deeper result, also proved in [HY], says that for a fixed $\lambda$, there is an open subset of closed points $s \in \operatorname{Spec} A$ such that equality holds in (1) for this $\lambda$. This relies on the same kind of arguments as in [Ha] and [MS], using the action of Frobenius on the de Rham complex, following [DI]. The key fact in the above conjecture is that we require the equality to hold for all $\lambda$ at the same time. We mention that these two known results generalize the fact that $\left(Y, \mathfrak{a}^{\lambda}\right)$ is Kawamata log terminal if and only if for an open (or just dense) set of closed points $S \subset \operatorname{Spec} A$, the pair $\left(Y_{s}, \mathfrak{a}_{s}^{\lambda}\right)$ is strongly $F$-regular for all $s \in S$. The following is our main result.

TheOrem 1.3. If Conjecture 1.1 holds, then Conjecture 1.2 holds as well.

It is easy to reduce the assertion in Conjecture 1.2 to the case when $Y$ is affine and $\mathfrak{a}$ is a principal ideal $(f)$. The usual approach for comparing the multiplier ideals of $\mathfrak{a}$ with the test ideals of a reduction $\bmod p$ of $\mathfrak{a}$ is to start with a log resolution of $\mathfrak{a}$. Our key point is to start instead by doing semistable reduction. This allows us to reduce at the end of the day to understanding a certain reduced divisor with simple normal crossings on a nonsingular variety.

One can formulate Conjecture 1.2 in a more general setting. For example, one can only assume that $Y$ is normal and Q-Gorenstein or, even more generally, work with a pair $(Y, D)$ such that $K_{Y}+D$ is Q-Cartier. Furthermore, one can start with several ideals $\mathfrak{a}_{1}, \ldots, \mathfrak{a}_{r}$ and consider mixed multiplier ideals and test ideals. However, our method based on semistable reduction does not allow us at present to handle these more general versions of Conjecture 1.2. 
The paper is organized as follows. In the next section we recall some general facts about $p$-linear maps of vector spaces over perfect fields and review the general setting for reducing from characteristic zero to positive characteristic. In Section 3 we recall the definition and some useful properties of multiplier ideals and test ideals. While in our main result we consider a nonsingular ambient variety, at an intermediate step we also need to work on a singular variety. Therefore, our treatment of multiplier ideals and test ideals in Section 3 is done in this general setting. In Section 4 we state and discuss a more general version of Conjecture 1.2. Section 5 is devoted to a discussion of Conjecture 1.1 and to several consequences that would be needed later. In Section 6 we prove our main result, showing that Conjecture 1.1 implies Conjecture 1.2.

\section{§2. A review of basic facts}

In this section we recall some well-known facts that will come up frequently during the rest of the paper. In particular, we discuss the general setting and set the notation for reduction $\bmod p$.

\section{1. $p$-linear maps on vector spaces}

Let $k$ be a perfect field of characteristic $p>0$, and let $V$ be a finitedimensional vector space over $k$. Let $\varphi: V \rightarrow V$ be a $p$-linear map, that is, a morphism of abelian groups such that $\varphi(a u)=a^{p} \varphi(u)$ for all $a \in k$ and $u \in V$. The following properties of such a map are well known (for a proof, see, e.g., [CL, lemme 3.3]).

The vector space $V$ can be uniquely decomposed as a direct sum of subspaces preserved by $\varphi, V=V_{\mathrm{ss}} \oplus V_{\text {nil }}$, where

(1) $\varphi$ is nilpotent on $V_{\text {nil }}$, that is, $\varphi^{N}=0$ for some $N$;

(2) $\varphi$ is bijective on $V_{\text {ss }}$.

One says that $\varphi$ is semisimple if $V=V_{\mathrm{ss}}$. This is equivalent with $\varphi$ being injective or, equivalently, surjective.

EXAMPLE 2.1. If $k$ is a finite field with $p^{e}$ elements, then $\varphi^{e}$ is a $k$-linear map. In this case $\varphi$ is semisimple if and only if $\varphi^{e}$ is an isomorphism.

If $\varphi$ is as above, and $k^{\prime}$ is a perfect field extension of $k$, then we get an induced $p$-linear map $\varphi^{\prime}: V^{\prime} \rightarrow V^{\prime}$, where $V^{\prime}=V \otimes_{k} k^{\prime}$. This is given by $\varphi^{\prime}(v \otimes \lambda)=\varphi(v) \otimes \lambda^{p}$. We have $V_{\mathrm{ss}}^{\prime}=V_{\mathrm{ss}} \otimes_{k} k^{\prime}$ and $V_{\text {nil }}^{\prime}=V_{\text {nil }} \otimes_{k} k^{\prime}$. In particular, $\varphi^{\prime}$ is semisimple if and only if $\varphi$ is semisimple. 
These considerations apply, in particular, if we take $k^{\prime}=\bar{k}$, an algebraic closure of $k$. If $\bar{\varphi}: \bar{V} \rightarrow \bar{V}$ is the induced $p$-linear map over $\bar{k}$, then $\bar{V}^{\bar{\varphi}=1}:=$

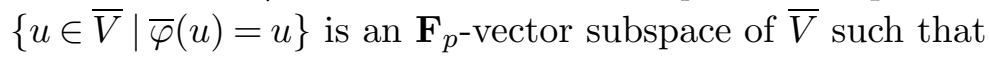

$$
\bar{V}_{\mathrm{ss}}=\bar{V}^{\bar{\varphi}=1} \otimes_{\mathbf{F}_{p}} \bar{k} .
$$

In particular, we have $\operatorname{dim}_{\mathbf{F}_{p}}\left(\bar{V}^{\bar{\varphi}=1}\right) \leq \operatorname{dim}_{k}(V)$, with equality if and only if $\varphi$ is semisimple.

Note that the morphism of abelian groups $1-\bar{\varphi}$ is surjective on $\bar{V}_{\text {ss }}$ by (2), and it is clearly bijective on $\bar{V}_{\text {nil }}$. In particular, $1-\bar{\varphi}$ is surjective, and its kernel is $\bar{V}^{\bar{\varphi}=1}$.

EXAMPLE 2.2. Let $X$ be a complete scheme of finite type over $k$. The absolute Frobenius morphism $F: X \rightarrow X$ is the identity on the underlying topological space, and the corresponding morphism of sheaves of rings $\mathcal{O}_{X} \rightarrow$ $\mathcal{O}_{X}$ is given by $u \rightarrow u^{p}$. Since $k$ is perfect, $F$ is a finite morphism. It induces a $p$-linear map $F: H^{i}\left(X, \mathcal{O}_{X}\right) \rightarrow H^{i}\left(X, \mathcal{O}_{X}\right)$ for every $i \geq 0$. After extending the scalars to an algebraic closure $\bar{k}$, we obtain the corresponding $p$-linear $\operatorname{map} F: H^{i}\left(X_{\bar{k}}, \mathcal{O}_{X_{\bar{k}}}\right) \rightarrow H^{i}\left(X_{\bar{k}}, \mathcal{O}_{X_{\bar{k}}}\right)$, where $X_{\bar{k}}=X \times_{\operatorname{Spec} k} \operatorname{Spec} \bar{k}$. (Note that in this case we still write $F$ instead of $\bar{F}$.)

On the other hand, we have the Artin-Schreyer sequence in the étale topology

$$
0 \rightarrow \mathbf{F}_{p} \rightarrow \mathcal{O}_{X_{\bar{k}}} \stackrel{1-F}{\rightarrow} \mathcal{O}_{X_{\bar{k}}} \rightarrow 0 .
$$

This induces exact sequences

$$
0 \rightarrow H_{\text {ét }}^{i}\left(X_{\bar{k}}, \mathbf{F}_{p}\right) \rightarrow H^{i}\left(X_{\bar{k}}, \mathcal{O}_{X_{\bar{k}}}\right) \stackrel{1-F}{\rightarrow} H^{i}\left(X_{\bar{k}}, \mathcal{O}_{X_{\bar{k}}}\right) \rightarrow 0
$$

for every $i \geq 0$. In particular, $F$ is semisimple on $H^{i}\left(X, \mathcal{O}_{X}\right)$ if and only if $\operatorname{dim}_{\mathbf{F}_{p}} H_{\text {ét }}^{i}\left(X_{\bar{k}}, \mathbf{F}_{p}\right)=h^{i}\left(X, \mathcal{O}_{X}\right)$.

REMARK 2.3. Let $\varphi: V \rightarrow V$ and $\psi: W \rightarrow W$ be $p$-linear maps as above. Note that we have induced $p$-linear maps on $V \oplus W$ and $V \otimes W$ and that $(V \oplus W)_{\mathrm{ss}}=V_{\mathrm{ss}} \oplus W_{\mathrm{ss}}$ and $(V \otimes W)_{\mathrm{ss}}=V_{\mathrm{ss}} \otimes W_{\mathrm{ss}}$.

Lemma 2.4. Let $\varphi: V \rightarrow V$ be a p-linear map as above.

(i) If $\varphi$ is semisimple, and if $W$ is a linear subspace of $V$ such that $\varphi(W) \subseteq$ $W$, then the induced $p$-linear maps on $W$ and $V / W$ are semisimple.

(ii) If we have an exact sequence $V^{\prime} \rightarrow V \rightarrow V^{\prime \prime}$ and $p$-linear maps $\varphi^{\prime}: V^{\prime} \rightarrow$ $V^{\prime}$ and $\varphi^{\prime \prime}: V^{\prime \prime} \rightarrow V^{\prime \prime}$ that are compatible with $\varphi$ in the obvious sense, and if $\varphi^{\prime}$ and $\varphi^{\prime \prime}$ are semisimple, then so is $\varphi$. 
Proof. If $\varphi$ is bijective, then clearly the induced map on $W$ is injective, and the induced map on $V / W$ is surjective. This implies the assertion in (i). In order to prove (ii), we use (i) to reduce to the case when we have a short exact sequence

$$
0 \rightarrow V^{\prime} \rightarrow V \rightarrow V^{\prime \prime} \rightarrow 0
$$

In this case $\varphi^{\prime}$ and $\varphi^{\prime \prime}$ being bijective implies that $\varphi$ is bijective by the 5-lemma.

\subsection{Reduction $\bmod p$}

We review the formalism for passing from characteristic zero to positive characteristic. Let $k$ be a fixed field of characteristic zero. Given a scheme $X$ of finite type over $k$, there is a subring $A \subset k$ of finite type over $\mathbf{Z}$, a scheme $X_{A}$ of finite type over $A$, and an isomorphism $X \simeq X_{A} \times_{\operatorname{Spec} A}$ Spec $k$. Note that we may always replace $A$ by $A_{a}$, for some nonzero $a \in A$, and $X_{A}$ by the corresponding open subscheme. It follows from generic flatness (see [Eis, Theorem 14.4]) that we may (and will) assume that $X_{A}$ is flat over $A$. We will refer to $X_{A}$ as a model of $X$ over $A$. If $A$ and $B$ are two such rings, and if $X_{A}$ and $X_{B}$ are models of $X$ over $A$ and $B$, respectively, then there is a subring $C$ of $k$ containing both $A$ and $B$, finitely generated over $\mathbf{Z}$, and an isomorphism

$$
X_{A} \times_{\operatorname{Spec} A} \operatorname{Spec} C \simeq X_{B} \times_{\operatorname{Spec} B} \operatorname{Spec} C
$$

compatible after base change to Spec $k$ with the defining isomorphisms for $X_{A}$ and $X_{B}$. Given a model $X_{A}$ for $X$ as above, and a point $s \in \operatorname{Spec} A$, we denote by $X_{s}$ the fiber of $X_{A}$ over $s$. This is a scheme of finite type over the residue field $k(s)$ of $s$. Note that if $s$ is a closed point, then $k(s)$ is a finite field.

We will consider properties $\mathcal{P}$ of schemes of finite type over finite fields such that, given a scheme $W$ of finite type over the finite field $k$ and a finite field extension $k^{\prime}$ of $k, \mathcal{P}(W)$ holds if and only if $\mathcal{P}\left(W \times{ }_{\text {Spec } k}\right.$ Spec $\left.k^{\prime}\right)$ holds. With $X_{A}$ as above, we say that $\mathcal{P}\left(X_{s}\right)$ holds for general closed points $s \in$ Spec $A$ if there is an open subset $U$ of Spec $A$ such that $\mathcal{P}\left(X_{s}\right)$ holds for all closed points $s \in U$. In this case, after replacing $A$ by a suitable localization $A_{a}$, we may assume that $\mathcal{P}\left(X_{s}\right)$ holds for all closed points $s$. We will often be interested in properties that are expected to hold only for a dense set of closed points $s \in \operatorname{Spec} A$. 
REMARK 2.5. With $\mathcal{P}$ as above, note that both conditions

(i) $\mathcal{P}\left(X_{s}\right)$ holds for general closed points $s \in \operatorname{Spec} A$,

(ii) $\mathcal{P}\left(X_{s}\right)$ holds for a dense set of closed points $s \in \operatorname{Spec} A$

are independent of the choice of a model. Indeed, if $\alpha: \operatorname{Spec} C \rightarrow \operatorname{Spec} A$ is induced by the inclusion $A \subset C$ of finitely generated $\mathbf{Z}$-algebras, then $\alpha$ takes closed points to closed points, and the image of $\alpha$ contains a (dense) open subset. Furthermore, the image or inverse image of a dense subset has the same property.

On the other hand, in order to show that (ii) above holds, it is enough to show that for every model $X_{A}$ there is at least one closed point $s \in \operatorname{Spec} A$ such that $\mathcal{P}\left(X_{s}\right)$ holds.

If $X_{A}$ is a model for $X$ as above, and if $\mathcal{F}$ is a coherent sheaf on $X$, then after possibly replacing $A$ by a larger ring we may assume that there is a coherent sheaf $\mathcal{F}_{A}$ on $X_{A}$ whose pullback to $X$ is isomorphic to $\mathcal{F}$. It follows from generic flatness that after replacing $A$ by some localization $A_{a}$, we may (and will) assume that $\mathcal{F}_{A}$ is flat over $A$. For a point $s \in \operatorname{Spec} A$, we denote by $\mathcal{F}_{s}$ the restriction of $\mathcal{F}_{A}$ to the fiber over $s$.

If $\varphi: \mathcal{F} \rightarrow \mathcal{G}$ is a morphism of coherent sheaves, after possibly enlarging $A$ we may assume that $f$ is induced by a morphism of sheaves $\varphi_{A}: \mathcal{F}_{A} \rightarrow \mathcal{G}_{A}$. In particular, for every point $s \in \operatorname{Spec} A$, we get an induced morphism $\varphi_{s}: \mathcal{F}_{s} \rightarrow$ $\mathcal{G}_{s}$. Since we may assume that $\operatorname{Coker}\left(\varphi_{A}\right)$ and $\operatorname{Im}\left(\varphi_{A}\right)$ are flat over $A$, it follows that we may assume that $\operatorname{Coker}\left(\varphi_{s}\right)=\operatorname{Coker}(\varphi)_{s}, \operatorname{Im}\left(\varphi_{s}\right)=\operatorname{Im}(\varphi)_{s}$, and $\operatorname{Ker}\left(\varphi_{s}\right)=\operatorname{Ker}(\varphi)_{s}$ for every point $s \in \operatorname{Spec} A$. In particular, if $\varphi$ is injective or surjective, then so are all $\varphi_{s}$. It follows easily from this that if $\mathcal{F}$ is an ideal, or if it is locally free, then so are all $\mathcal{F}_{s}$ (as well as $\mathcal{F}_{A}$ ).

Given a morphism $f: X \rightarrow Y$ of schemes of finite type over $k$, and models $X_{A}$ and $Y_{B}$ of $X$ and $Y$, respectively, after possibly enlarging both $A$ and $B$ we may assume that $A=B$ and that $f$ is induced by a morphism $f_{A}: X_{A} \rightarrow Y_{A}$ of schemes over $A$. If $s \in \operatorname{Spec} A$ is a point, then we get a corresponding morphism $f_{s}: X_{s} \rightarrow Y_{s}$ of schemes over $k(s)$. If $f$ is a closed (open) immersion, finite or projective, then we may assume that the same holds for $f_{A}$. In particular, the same will hold for all $f_{s}$.

Suppose now that $f: X \rightarrow Y$ is a proper morphism and that $\mathcal{F}$ is a coherent sheaf on $X$. If $f_{A}: X_{A} \rightarrow Y_{A}$ and $\mathcal{F}_{A}$ are as above, arguing as in [Hart, Section III.12] one can show that $\mathcal{F}_{A}$ satisfies generic base change. In other words, after replacing $A$ by $A_{a}$ for some nonzero $a \in A$, we may assume that 
for all $s \in \operatorname{Spec} A$, the canonical morphism

$$
\left(R^{i}\left(f_{A}\right)_{*}\left(\mathcal{F}_{A}\right)\right)_{s} \rightarrow R^{i}\left(f_{s}\right)_{*}\left(\mathcal{F}_{s}\right)
$$

is an isomorphism.

Given a model $X_{A}$ of $X$, it is easy to deduce from Noether's normalization theorem that all fibers of $X_{A} \rightarrow \operatorname{Spec} A$ have dimension $\leq \operatorname{dim}(X)$. It follows from the Jacobian criterion for smoothness that if $X$ is an irreducible regular scheme, then we may assume that $X_{A}$ is smooth over $\operatorname{Spec} A$ of relative dimension equal to $\operatorname{dim}(X)$. In particular, $X_{s}$ is smooth over $k(s)$ for every point $s \in \operatorname{Spec} A$. In general, $X_{s}$ might not be connected; however, if we assume that $k$ is algebraically closed, then $X_{s}$ will be connected, since the generic fiber of $X_{A}$ over $\operatorname{Spec} A$ is geometrically connected.

For simplicity, from now on we assume that $k$ is algebraically closed. Suppose that $Y$ is an arbitrary reduced scheme over $k$, and let us consider a resolution of singularities of $Y$, that is, a projective birational morphism $f: X \rightarrow Y$, with $X$ regular. We may choose a morphism of models $f_{A}: X_{A} \rightarrow$ $Y_{A}$ that is projective, birational, and with $X_{A}$ smooth over $\operatorname{Spec} A$. We may also assume that $\operatorname{Spec} A$ is smooth over Spec $\mathbf{Z}$. Since $\mathcal{O}_{Y} \hookrightarrow f_{*}\left(\mathcal{O}_{X}\right)$, we may assume that $\mathcal{O}_{Y_{A}} \hookrightarrow\left(f_{A}\right)_{*}\left(\mathcal{O}_{X_{A}}\right)$. In particular, $Y_{A}$ is reduced. Furthermore, by generic base change we may assume that $\mathcal{O}_{Y_{s}} \hookrightarrow\left(f_{s}\right)_{*}\left(\mathcal{O}_{X_{s}}\right)$ for every $s \in \operatorname{Spec} A$. In particular, $Y_{s}$ is reduced, and if $Y$ is irreducible, then so are all $Y_{s}$. (Here we make use of the assumption that $k$ is algebraically closed.) We also see that $\operatorname{dim}\left(Y_{s}\right)=\operatorname{dim}(Y)$ for all $s$, since we know this property for $X$. Similarly, if $Y$ is normal, then $\mathcal{O}_{Y}=f_{*}\left(\mathcal{O}_{X}\right)$, and arguing as above we may assume that $Y_{A}$ and all $Y_{s}$ are normal.

If $D=a_{1} D_{1}+\cdots+a_{r} D_{r}$ is a Weil divisor on $Y$, then we may assume that we have prime divisors $\left(D_{i}\right)_{A}$ on $Y_{A}$, and let $D_{A}:=\sum_{i} a_{i}\left(D_{i}\right)_{A}$. After possibly replacing $A$ by a localization $A_{a}$, we may assume that for every $s \in \operatorname{Spec} A$ the fiber $\left(D_{i}\right)_{s}$ is a prime divisor on $Y_{s}$, and we get the divisor $D_{s}=\sum_{i} a_{i}\left(D_{i}\right)_{s}$.

In particular, if $Y$ is irreducible and normal, we may consider $K_{Y}$, a Weil divisor unique up to linear equivalence, whose restriction to the nonsingular locus $Y_{\mathrm{sm}}$ is a divisor corresponding to $\omega_{Y_{\mathrm{sm}}}$. We write $K_{Y_{A}}$ for $\left(K_{Y}\right)_{A}$. If $U=Y_{\mathrm{sm}}$, then we may assume that the corresponding open subset $U_{A} \subset$ $Y_{A}$ is smooth over $A$ and that $K_{Y_{A}}$ is a divisor whose restriction to $U_{A}$ corresponds to $\Omega_{U_{A} / A}^{n}$, where $n=\operatorname{dim}(Y)$. We may therefore assume that for every $s \in \operatorname{Spec} A$, the restriction of $K_{Y_{A}}$ to $Y_{s}$ gives a canonical divisor $K_{Y_{s}}$. 


\section{$\S 3 . \quad$ Test ideals and multiplier ideals}

\subsection{Multiplier ideals}

We start by recalling the definition of multiplier ideals. (For details, basic properties, and further results, see [Laz].) Let $k$ be an algebraically closed field of characteristic zero, and let $Y$ be an irreducible normal scheme of finite type over $k$. We consider a Weil divisor $D$ on $Y$ such that $K_{Y}+D$ is Cartier.* Given a nonzero ideal $\mathfrak{a}$ on $Y$, we define the multiplier ideals $\mathcal{J}\left(Y, D, \mathfrak{a}^{\lambda}\right)$ for $\lambda \in \mathbf{R}_{\geq 0}$ as follows.

Recall first that given any birational morphism $\pi: X \rightarrow Y$, with $X$ normal, there is a unique divisor $D_{X}$ on $X$ with the following two properties:

(i) $K_{X}+D_{X}$ is linearly equivalent with $\pi^{*}\left(K_{Y}+D\right)$ (hence, in particular, it is Cartier);

(ii) for every nonexceptional prime divisor $T$ on $X$, its coefficient in $D_{X}$ is equal to its coefficient in the strict transform $\widetilde{D}$ of $D$.

Note that $D_{X}$ is supported on $\widetilde{D}+\operatorname{Exc}(\pi)$, where $\operatorname{Exc}(\pi)$ is the exceptional locus of $\pi$.

Suppose now that $\pi: X \rightarrow Y$ is a $\log$ resolution of the triple $(Y, D, \mathfrak{a})$. This means that $\pi$ is projective and birational, $X$ is nonsingular, $\mathfrak{a} \cdot \mathcal{O}_{X}=$ $\mathcal{O}_{X}(-G)$ for a divisor $G, \operatorname{Exc}(\pi)$ is a divisor, and $E:=\widetilde{D}+\operatorname{Exc}(\pi)+G$ has simple normal crossings. With this notation, we have

$$
\mathcal{J}\left(X, D, \mathfrak{a}^{\lambda}\right):=\pi_{*} \mathcal{O}_{X}\left(-D_{X}-\lfloor\lambda \cdot G\rfloor\right) .
$$

Recall that if $T=\sum_{i} b_{i} T_{i}$ is an $\mathbf{R}$-divisor, then $\lfloor T\rfloor:=\sum_{i}\left\lfloor b_{i}\right\rfloor T_{i}$, where $\left\lfloor b_{i}\right\rfloor$ is the largest integer $\leq b_{i}$. When $\mathfrak{a}=(f)$ is a principal ideal, then we simply write $\mathcal{J}\left(X, D, f^{\lambda}\right)$. Note that $\mathcal{J}\left(X, D, \mathfrak{a}^{\lambda}\right)$ is in general only a fractional ideal. However, if $D$ is effective, then all components of $D_{X}$ with negative coefficient are exceptional. Therefore, in this case $\mathcal{J}\left(X, D, \mathfrak{a}^{\lambda}\right)$ is an ideal.

It is a basic fact that the above definition is independent of resolution. It follows from (3) that $\mathcal{J}\left(Y, D, \mathfrak{a}^{\lambda}\right) \subseteq \mathcal{J}\left(Y, D, \mathfrak{a}^{\mu}\right)$ if $\lambda>\mu$. Furthermore, given any $\lambda \geq 0$, there is $\varepsilon>0$ such that $\mathcal{J}\left(Y, D, \mathfrak{a}^{\lambda}\right)=\mathcal{J}\left(Y, D, \mathfrak{a}^{\mu}\right)$ for all $\mu$ with $\lambda \leq \mu \leq \lambda+\varepsilon$. One says that $\lambda>0$ is a jumping number of $(Y, D, \mathfrak{a})$ if $\mathcal{J}\left(Y, D, \mathfrak{a}^{\lambda}\right) \neq \mathcal{J}\left(Y, D, \mathfrak{a}^{\mu}\right)$ for every $\mu<\lambda$. Note that if we write $G=$ $\sum_{i=1}^{N} b_{i} E_{i}$, then for every jumping number $\lambda$ we must have

$$
\lambda b_{i} \in \mathbf{Z} \quad \text { for some } i \leq N \text { with } b_{i} \neq 0 .
$$

\footnotetext{
*One can assume that $D$ is just a $\mathbf{Q}$-divisor such that some multiple of $K_{Y}+D$ is Cartier; however, we will not need this level of generality.
} 
(If $\lambda$ satisfies this property, we call it a candidate jumping number.) In particular, the set of jumping numbers of $(X, D, \mathfrak{a})$ is a discrete subset of $\mathbf{Q}_{>0}$.

We now recall a few properties of multiplier ideals that will come up later. The following result is [Laz, Theorem 9.2.33]. The proof uses the definition of multiplier ideals and the independence of resolutions. (While the statement therein requires the varieties to be nonsingular, the same proof works in the general setting.)

Proposition 3.1. If $\pi: X \rightarrow Y$ is any projective, birational morphism, with $X$ normal, and if $\mathfrak{a}^{\prime}=\mathfrak{a} \cdot \mathcal{O}_{X}$, then for every $\lambda \in \mathbf{R}_{\geq 0}$ we have

$$
\mathcal{J}\left(Y, D, \mathfrak{a}^{\lambda}\right)=\pi_{*} \mathcal{J}\left(X, D_{X},\left(\mathfrak{a}^{\prime}\right)^{\lambda}\right) .
$$

We now consider a finite surjective morphism $\mu: Y^{\prime} \rightarrow Y$, with $Y^{\prime}$ normal and irreducible, and we put $\mathfrak{a}^{\prime}=\mathfrak{a} \cdot \mathcal{O}_{Y^{\prime}}$. In this case there is an open subset $U \subseteq Y$ such that $\operatorname{codim}(Y \backslash U, Y) \geq 2$, and both $U$ and $V=\varphi^{-1}(U)$ are nonsingular (e.g., one can take $U=Y_{\mathrm{sm}} \backslash \mu\left(Y^{\prime} \backslash Y_{\mathrm{sm}}^{\prime}\right)$. In this case, both $K_{V / U}$ and $\mu^{*}\left(\left.D\right|_{U}\right)$ are well-defined divisors on $V$. We denote by $D_{Y^{\prime}}$ the unique Weil divisor on $Y^{\prime}$ whose restriction to $V$ is $\mu^{*}\left(\left.D\right|_{U}\right)-K_{V / U}$. Note that $K_{Y^{\prime}}+D_{Y^{\prime}}$ is linearly equivalent with $\mu^{*}\left(K_{Y}+D\right)$; hence, in particular, it is Cartier. For an integral scheme $W$, we denote by $K(W)$ the function field of $W$.

Proposition 3.2. With the above notation, for every $\lambda \in \mathbf{R}_{\geq 0}$ we have

$$
\mathcal{J}\left(Y, D, \mathfrak{a}^{\lambda}\right)=\mu_{*} \mathcal{J}\left(Y^{\prime}, D_{Y^{\prime}},\left(\mathfrak{a}^{\prime}\right)^{\lambda}\right) \cap K(Y) .
$$

Proof. If both $Y$ and $Y^{\prime}$ are nonsingular, then the result is [Laz, Theorem 9.5.42]. Note that the result therein only requires $\mu$ to be generically finite. The singular case is an easy consequence: if $X \rightarrow Y$ is a resolution of singularities, and if $X^{\prime} \rightarrow X \times_{Y} Y^{\prime}$ is a resolution of singularities of the irreducible component dominating $Y^{\prime}$, we get a commutative diagram

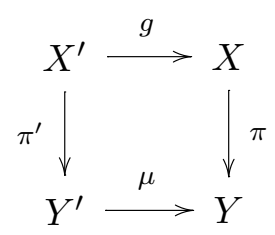

with $\pi$ and $\pi^{\prime}$ projective and birational, and $g$ generically finite. Applying [Laz, Theorem 9.5.42] to $g$, and Proposition 3.1 to $\pi$ and $\pi^{\prime}$, we deduce the equality in the proposition. 
Remark 3.3. Note that if the divisor $D$ in Proposition 3.2 is effective, then the proposition implies that

$$
\mathcal{J}\left(Y, D, \mathfrak{a}^{\lambda}\right)=\mu_{*} \mathcal{J}\left(Y^{\prime}, D_{Y^{\prime}},\left(\mathfrak{a}^{\prime}\right)^{\lambda}\right) \cap \mathcal{O}_{Y}
$$

The following statement follows directly from the definition of multiplier ideals and the projection formula (see [Laz, Proposition 9.2.31]).

Proposition 3.4. Let $(Y, D, \mathfrak{a})$ be as above, and suppose that $D^{\prime}$ is a Cartier divisor on $Y$. For every $\lambda \geq 0$ we have

$$
\mathcal{J}\left(Y, D+D^{\prime}, \mathfrak{a}^{\lambda}\right)=\mathcal{J}\left(Y, D, \mathfrak{a}^{\lambda}\right) \cdot \mathcal{O}_{Y}\left(-D^{\prime}\right) .
$$

The following result is [Laz, Proposition 9.2.28]. It is a consequence of Bertini's theorem.

Proposition 3.5. Suppose that $Y=\operatorname{Spec} R$ is affine, $\mathfrak{a}=\left(h_{1}, \ldots, h_{m}\right)$, and $d$ is a positive integer. If $g_{1}, \ldots, g_{d}$ are general linear combinations of the $h_{i}$ with coefficients in $k$, and if $g=\prod_{i=1}^{d} g_{i}$, then

$$
\mathcal{J}\left(Y, D, \mathfrak{a}^{\lambda}\right)=\mathcal{J}\left(Y, D, g^{\lambda / d}\right)
$$

for every $\lambda<d$.

We end this section with a statement of Skoda's theorem for multiplier ideals on singular varieties (for a proof, see [LLS, Corollary 1.4]). Note, however, that in this paper we will need only the case when $X$ is nonsingular. A proof in this case can be found in [Laz, Section 11.1.A].

Proposition 3.6. Let $(Y, D, \mathfrak{a})$ be as above. If $\mathfrak{a}$ can be locally generated by $m$ sections, then

$$
\mathcal{J}\left(Y, D, \mathfrak{a}^{\lambda}\right)=\mathfrak{a} \cdot \mathcal{J}\left(Y, D, \mathfrak{a}^{\lambda-1}\right)
$$

for every $\lambda \geq m$.

\subsection{Reduction $\bmod p$ of multiplier ideals}

Suppose now that $Y, D$, and $\mathfrak{a}$ are as in Section 3.1, and let us consider a model $Y_{A}$ of $Y$ over a finitely generated $\mathbf{Z}$-subalgebra $A$ of $k$. We follow the notation introduced in Section 2.2. We may assume that we have a Weil divisor $D_{A}$ on $Y_{A}$ and a sheaf of ideals $\mathfrak{a}_{A}$ on $Y_{A}$ that give models for $D$ and $\mathfrak{a}$. Let us fix now a $\log$ resolution $\pi: X \rightarrow Y$ of $(Y, D, \mathfrak{a})$. We may assume that this is induced by a projective birational morphism $X_{A} \rightarrow Y_{A}$, with $X_{A}$ smooth over $A$. 
Note that since $K_{Y}+D$ is Cartier, we may assume that $K_{Y_{A}}+D_{A}$ is Cartier, and that all $K_{Y_{s}}+D_{s}$ are Cartier, for $s \in \operatorname{Spec} A$. The divisor $D_{X_{A}}$ on $X_{A}$ that induces $D_{X}$ satisfies analogous properties to properties (i) and (ii) stated in Section 3.1 for $D_{X}$. Furthermore, for every $s \in \operatorname{Spec} A$, the restriction $D_{X_{s}}$ of $D_{X_{A}}$ to $X_{s}$ satisfies

(i) the Cartier divisors $K_{X_{s}}+D_{X_{s}}$ and $\pi_{s}^{*}\left(K_{Y_{s}}+D_{s}\right)$ are linearly equivalent;

(ii) for every nonexceptional prime divisor $T$ on $X_{s}$, its coefficient in $D_{X_{s}}$ is equal to its coefficient in the proper transform $\widetilde{D_{s}}$ of $D_{s}$.

We may assume that $\mathfrak{a}_{A} \cdot \mathcal{O}_{X_{A}}=\mathcal{O}_{X_{A}}\left(-G_{A}\right)$ for a divisor $G_{A}$ on $X_{A}$, and that $\operatorname{Exc}\left(\pi_{A}\right)$ is a divisor. Furthermore, we may assume that we have a divisor $E_{A}=\sum_{i=1}^{N}\left(E_{i}\right)_{A}$ on $X_{A}$ such that every intersection $\left(E_{i_{1}}\right)_{A} \cap$ $\cdots \cap\left(E_{i_{\ell}}\right)_{A}$ is smooth over $A$, and such that $G_{A}, \operatorname{Exc}\left(\pi_{A}\right)$, and $D_{X_{A}}$ are supported on $\operatorname{Supp}\left(E_{A}\right)$. We deduce that for every $s \in \operatorname{Spec} A$, the induced morphism $\pi_{s}: X_{s} \rightarrow Y_{s}$ gives a log resolution of $\left(Y_{s}, D_{s}, \mathfrak{a}_{s}\right)$.

Suppose now that $m$ is a positive integer such that $\mathfrak{a}$ can be generated locally by $m$ sections. Recall that in this case we have by Proposition 3.6 $\mathcal{J}\left(Y, D, \mathfrak{a}^{\lambda}\right)=\mathfrak{a} \cdot \mathcal{J}\left(Y, D, \mathfrak{a}^{\lambda-1}\right)$ for every $\lambda \geq m$. This allows us to focus on exponents $<m$ in defining $\mathcal{J}\left(Y, D, \mathfrak{a}^{\lambda}\right)_{s}$ and then to extend the definition by putting $\mathcal{J}\left(Y, D, \mathfrak{a}^{\lambda}\right)_{s}=\mathfrak{a}_{s} \cdot \mathcal{J}\left(Y, D, \mathfrak{a}^{\lambda-1}\right)_{s}$ for $\lambda \geq m$.

For $\lambda<m$, we have the ideal $\left(\pi_{A}\right)_{*} \mathcal{O}_{X_{A}}\left(-\left(D_{X}\right)_{A}-\left\lfloor\lambda G_{A}\right\rfloor\right)$ on $Y_{A}$ that gives a model of $\mathcal{J}\left(Y, D, \mathfrak{a}^{\lambda}\right)$. By generic base change, we may assume that for every $s \in \operatorname{Spec} A$, the induced ideal $\mathcal{J}\left(Y, D, \mathfrak{a}^{\lambda}\right)_{s}$ is the ideal $\left(\pi_{s}\right)_{*} \mathcal{O}_{X_{s}}\left(-D_{X_{s}}-\left\lfloor\lambda G_{s}\right\rfloor\right)$. Indeed, note that we only have to consider finitely many ideals, corresponding to the candidate jumping numbers as in (4) that are $<m$. We mention that if we consider the above construction starting with a different $\log$ resolution of $(Y, D, \mathfrak{a})$, then there is an open subset of Spec $A$ such that for every $s$ in this subset, the two definitions of the ideals $\mathcal{J}\left(Y, D, \mathfrak{a}^{\lambda}\right)_{s}$ coincide.

\subsection{Test ideals}

In this section we work over a perfect field $L$ of positive characteristic $p$. (In the case of interest for us, $L$ will always be a finite field.) In this case, the test ideals of Hara and Yoshida [HY] admit a simpler description, due to Schwede [Sch], that completely avoids tight closure theory. Our main reference here is $[\mathrm{ST}]$, though for some of the proofs the reader will need to consult the references given therein. 
Before giving the definition of test ideals, we review a fundamental map in positive characteristic. Suppose that $Y$ is a smooth connected scheme over $L$ of dimension $n$. Let $\Omega_{Y / L}^{\bullet}$ be the de Rham complex on $Y$. If $F$ denotes the absolute Frobenius morphism on $Y$, then the Cartier isomorphism is a graded $\mathcal{O}_{Y}$-linear isomorphism $C_{Y}: \bigoplus_{i} \mathcal{H}^{i}\left(F_{*}\left(\Omega_{Y / L}^{\bullet}\right)\right) \rightarrow \bigoplus_{i} \Omega_{Y / L}^{i}$ (see [DI] for a description and proof). In particular, we get a surjection

$$
F_{*} \omega_{Y}=F_{*} \Omega_{Y / L}^{n} \rightarrow \mathcal{H}^{n}\left(F_{*}\left(\Omega_{Y / L}^{\bullet}\right)\right) \stackrel{C_{Y}}{\rightarrow} \omega_{Y},
$$

which we denote by $t_{Y}$. Iterating this map $e$ times gives $t_{Y}^{e}: F_{*}^{e} \omega_{Y} \rightarrow \omega_{Y}$.

If $f$ and $w$ are local sections of $\mathcal{O}_{Y}$ and $\omega_{Y}$, respectively, then $t_{Y}((1 / f) w)=(1 / f) t_{Y}\left(f^{p-1} w\right)$. This shows that for every effective divisor $D$ on $Y$, we have an induced map

$$
t_{Y, D}: F_{*}\left(\omega_{Y}(D)\right) \rightarrow \omega_{Y}(D)
$$

compatible with the previous one via the inclusion $\omega_{Y} \hookrightarrow \omega_{Y}(D)$. The same remark applies to the maps $t_{Y}^{e}$. If $D$ is not necessarily effective, then $t_{Y, D}$ is still well defined, but its image lies in the sheaf $\omega_{Y} \otimes K(Y)$ of rational $n$-forms on $Y$.

The map $t_{Y}: F_{*}\left(\omega_{Y}\right) \rightarrow \omega_{Y}$ can be described around a closed point $y \in$ $Y$, as follows. Let us choose a system of coordinates $u_{1}, \ldots, u_{n}$ at $y$ (i.e., a regular system of parameters of $\mathcal{O}_{Y, y}$ ). We may assume that we have an affine open neighborhood $U$ of $y$ such that $u_{i} \in \mathcal{O}_{Y}(U)$ for all $i$, and that $d u=d u_{1} \wedge \cdots \wedge d u_{n}$ gives a basis of $\left.\omega_{Y}\right|_{U}$. Furthermore, the residue field of $\mathcal{O}_{Y, y}$ is finite over the perfect field $L$; hence, it is perfect; and since the $u_{i}$ give a regular system of parameters at $y$, we may assume that $\mathcal{O}_{Y}(U)$ is free over $\mathcal{O}_{Y}(U)^{p}$, with a basis given by

$$
\left\{u_{1}^{i_{1}} \cdots u_{n}^{i_{n}} \mid 0 \leq i_{j} \leq p-1 \text { for } 1 \leq j \leq n\right\} .
$$

In this case $\left.t_{Y}\right|_{U}$ is characterized by the fact that $t_{Y}\left(h^{p} w\right)=h \cdot t_{Y}(w)$ for every $h \in \mathcal{O}_{Y}(U)$, and for every $i_{j}$ with $0 \leq i_{j} \leq p-1$ we have

$$
t_{Y}\left(u_{1}^{i_{1}} \cdots u_{n}^{i_{n}} d u\right)= \begin{cases}d u, & \text { if } i_{j}=p-1 \text { for all } j \\ 0, & \text { otherwise }\end{cases}
$$

The map $t_{Y}$ is functorial in the following sense. Consider a morphism $\pi: X \rightarrow Y$ of smooth schemes over $L$. For every $i$ we have a commutative 
diagram involving the respective Cartier isomorphisms

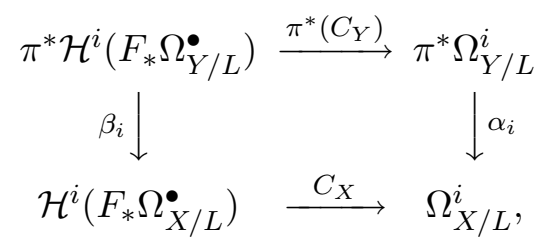

where $\alpha_{i}$ is given by pulling back forms, while $\beta_{i}$ is obtained as the composition

$$
\pi^{*} \mathcal{H}^{i}\left(F_{*} \Omega_{Y / L}^{\bullet}\right) \rightarrow \mathcal{H}^{i}\left(\pi^{*}\left(F_{*} \Omega_{Y / L}^{\bullet}\right)\right) \rightarrow \mathcal{H}^{i}\left(F_{*} \pi^{*} \Omega_{Y / L}^{\bullet}\right) \rightarrow \mathcal{H}^{i}\left(F_{*} \Omega_{X / L}^{\bullet}\right)
$$

If, in addition, $\pi$ is a proper birational map, $D$ is an effective divisor on $Y$, and $D_{X}$ is the divisor on $X$ defined as in Section 3.1, we get an induced commutative diagram relating the two trace maps

$$
\begin{gathered}
\pi^{*}\left(F_{*}^{e}\left(\omega_{Y}(D)\right)\right) \stackrel{\pi^{*}\left(t_{Y, D}^{e}\right)}{\longrightarrow} \pi^{*}\left(\omega_{Y}(D)\right) \\
F_{*}^{e}\left(\omega_{X}\left(D_{X}\right)\right) \stackrel{t_{X, D_{X}}^{e}}{\longrightarrow} \omega_{X}\left(D_{X}\right) \otimes K(X),
\end{gathered}
$$

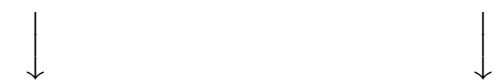

where the right vertical map is obtained by composing the isomorphism $\psi: \pi^{*}\left(\omega_{Y}(D)\right) \rightarrow \omega_{X}\left(D_{X}\right)$ with the inclusion $\omega_{X}\left(D_{X}\right) \hookrightarrow \omega_{X}\left(D_{X}\right) \otimes K(X)$, and the left vertical map is given by the composition

$$
\pi^{*}\left(F_{*}^{e}\left(\omega_{Y}(D)\right)\right) \longrightarrow F_{*}^{e}\left(\pi^{*}\left(\omega_{Y}(D)\right)\right) \stackrel{F_{*}^{e}(\psi)}{\longrightarrow} F_{*}^{e}\left(\omega_{X}\left(D_{X}\right)\right) .
$$

Note that while the bottom horizontal map in (7) does not necessarily land in $\omega_{X}\left(D_{X}\right)$ (since in general $D_{X}$ is not effective), the composition of the maps in (7) has this property.

Suppose now that $Y$ is a normal, irreducible scheme over $L$, of dimension $n$. We fix an effective Weil divisor $D$ on $Y$ such that $K_{Y}+D$ is Cartier. (Note that in [ST] one works in a more general framework, which complicates some of the definitions; for the sake of simplicity, we only give the definitions in the setting that we will need.) We claim that to $D$ and to every $e \geq 1$ we can naturally associate an $\mathcal{O}_{Y}$-linear map

$$
\varphi_{D}^{(e)}: F_{*}^{e} \mathcal{O}_{Y}\left(\left(1-p^{e}\right)\left(K_{Y}+D\right)\right) \rightarrow \mathcal{O}_{Y}
$$

Indeed, in order to define $\varphi_{D}^{(e)}$, it is enough to do it on the complement of a closed subset of codimension $\geq 2$. Therefore we may assume that $Y$ is 
smooth over $L$. In this case $\varphi_{D}^{(e)}$ is obtained by tensoring $t_{Y, D}^{e}: F_{*}^{e}\left(\omega_{Y}(D)\right) \rightarrow$ $\omega_{Y}(D)$ by $\omega_{Y}^{-1}(-D)$ and using the projection formula for $F^{e}$. Note that if $D$ is not necessarily effective, then we may still define as above $\varphi_{D}^{(e)}$, but its image will be a fractional ideal on $Y$, not necessarily contained in $\mathcal{O}_{Y}$.

If $\pi: X \rightarrow Y$ is a proper, birational morphism of schemes, with $X$ smooth, then we have as in Section 3.1 a unique divisor $D_{X}$ on $X$ such that $K_{X}+D_{X}$ is linearly equivalent to $\pi^{*}\left(K_{Y}+D\right)$, and such that $D_{X}$ agrees along the nonexceptional divisors of $\pi$ with the proper transform of $D$. In this case, we claim that the commutative diagram (7) induces a commutative diagram

$$
\begin{array}{cc}
\pi^{*} F_{*}^{e} \mathcal{O}_{Y}\left(\left(1-p^{e}\right)\left(K_{Y}+D\right)\right) & \stackrel{\pi^{*}\left(\varphi_{D}^{(e)}\right)}{\longrightarrow} \pi^{*} \mathcal{O}_{Y} \\
\downarrow & \downarrow \\
F_{*}^{e} \mathcal{O}_{X}\left(\left(1-p^{e}\right)\left(K_{X}+D_{X}\right)\right) & \stackrel{\varphi_{D_{X}}^{(e)}}{\longrightarrow} K(X),
\end{array}
$$

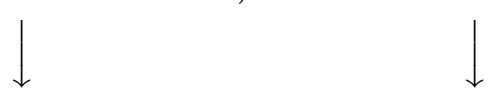

where the right vertical map is given by $\pi^{*}\left(\mathcal{O}_{Y}\right) \simeq \mathcal{O}_{X} \hookrightarrow K(X)$. This follows when $Y$ is smooth, too, using the commutativity of $(7)$. In the general case, note that $(9)$ corresponds by the adjointness of $\left(\pi^{*}, \pi_{*}\right)$ to the diagram

$$
\begin{array}{cc}
F_{*}^{e} \mathcal{O}_{Y}\left(\left(1-p^{e}\right)\left(K_{Y}+D\right)\right) & \stackrel{\varphi_{D}^{(e)}}{\longrightarrow} \mathcal{O}_{Y} \\
F_{*}^{e}(\rho) \downarrow & \downarrow \\
\pi_{*} F_{*}^{e} \mathcal{O}_{X}\left(\left(1-p^{e}\right)\left(K_{X}+D_{X}\right)\right) & \stackrel{\pi_{*}\left(\varphi_{D_{X}}^{(e)}\right)}{\longrightarrow} K(Y),
\end{array}
$$

where $\rho: \mathcal{O}_{Y}\left(\left(1-p^{e}\right)\left(K_{Y}+D\right)\right) \rightarrow \pi_{*} \mathcal{O}_{X}\left(\left(1-p^{e}\right)\left(K_{X}+D_{X}\right)\right)$ is the canonical isomorphism given by pullback of sections. In order to check the commutativity of (10) we may restrict to the complement of a codimension $\geq 2$ closed subset, and therefore we may assume that both $X$ and $Y$ are smooth, in which case, as we have mentioned, (9) hence also (10) is commutative. Note also that since (10) is commutative and the left vertical map is an isomorphism, the image of $\pi_{*}\left(\varphi_{D_{X}}^{(e)}\right)$ is contained in $\mathcal{O}_{Y}=\pi_{*}\left(\mathcal{O}_{X}\right)$.

ExAmPle 3.7. Suppose that $Y$ is nonsingular and that $D=a_{1} E_{1}+\cdots+$ $a_{r} E_{r}$ is a not necessarily effective simple normal crossings divisor on $Y$. If $\mathfrak{b}=\mathcal{O}_{Y}\left(-D^{\prime}\right)$, where $D^{\prime}=\sum_{i=1}^{r} b_{i} E_{i}$ is effective, then

$$
\varphi_{D}^{(e)}\left(F_{*}^{e}\left(\mathfrak{b} \cdot \mathcal{O}_{Y}\left(\left(1-p^{e}\right)\left(K_{Y}+D\right)\right)\right)\right)=\mathcal{O}_{Y}(-D-F),
$$


where $F=\sum_{i=1}^{r} c_{i} E_{i}$, with $c_{i}=\left\lfloor\left(b_{i}-a_{i}\right) / p^{e}\right\rfloor$ for every $i$. This description follows easily from the description in coordinates of the map $t_{Y, D}^{e}$.

We can now recall the definition of the test ideal $\tau\left(Y, D, \mathfrak{a}^{\lambda}\right)$, where $(Y, D)$ is as above (with $D$ effective), $\mathfrak{a}$ is a nonzero ideal on $Y$, and $\lambda$ is a nonnegative real number. One shows that there is a unique minimal nonzero coherent ideal sheaf $J$ on $Y$ such that for every $e$ we have

$$
\varphi_{D}^{(e)}\left(F_{*}^{e}\left(\mathfrak{a}^{\left\lceil\lambda\left(p^{e}-1\right)\right\rceil} J \cdot \mathcal{O}_{Y}\left(\left(1-p^{e}\right)\left(K_{Y}+D\right)\right)\right)\right) \subseteq J .
$$

This is the test ideal $\tau\left(Y, D, \mathfrak{a}^{\lambda}\right)$. Here $\lceil u\rceil$ denotes the smallest integer $\geq u$. When $\mathfrak{a}=(f)$ is a principal ideal, we simply write $\tau\left(Y, D, f^{\lambda}\right)$.

Proposition 3.8. If $(Y, D)$ is as above, and $d$ is a positive integer, then

$$
\tau\left(Y, D,\left(\mathfrak{a}^{d}\right)^{\lambda}\right) \subseteq \tau\left(Y, D, \mathfrak{a}^{d \lambda}\right) \quad \text { for every } \lambda \in \mathbf{R}_{\geq 0} .
$$

Proof. For every $e$ we have $d\left\lceil\lambda\left(p^{e}-1\right)\right\rceil \geq\left\lceil d \lambda\left(p^{e}-1\right)\right\rceil$. It follows that if $J$ satisfies (11) with $\mathfrak{a}^{\left\lceil\lambda\left(p^{e}-1\right)\right\rceil}$ replaced by $\mathfrak{a}^{\left\lceil d \lambda\left(p^{e}-1\right)\right\rceil}$, then it also satisfies (11) with $\mathfrak{a}^{\left\lceil\lambda\left(p^{e}-1\right)\right\rceil}$ replaced by $\mathfrak{a}^{d\left\lceil\lambda\left(p^{e}-1\right)\right\rceil}$. The assertion in the proposition now follows from the minimality in the definition of $\tau\left(Y, D,\left(\mathfrak{a}^{d}\right)^{\lambda}\right)$.

Remark 3.9. In fact, the inclusion in Proposition 3.8 is an equality. We leave to the interested reader the task of checking the reverse inclusion, that we will not need. See [BMS, Corollary 2.15] for a proof in the case when $Y$ is nonsingular.

In order to describe $\tau\left(Y, D, \mathfrak{a}^{\lambda}\right)$, it is enough to do it when $Y=\operatorname{Spec} R$ is affine. In this case one can show (see [ST, Lemma 6.4]) that there is a nonzero $c \in R$ such that for every nonzero $g \in R$, there is $e \geq 1$ such that

$$
c \in \varphi_{D}^{(e)}\left(F_{*}^{e}\left(\mathfrak{a}^{\left\lceil\lambda\left(p^{e}-1\right)\right\rceil} g \cdot \mathcal{O}_{Y}\left(\left(1-p^{e}\right)\left(K_{Y}+D\right)\right)\right)\right) .
$$

In this case, it is not hard to see that

$$
\tau\left(Y, D, \mathfrak{a}^{\lambda}\right)=\sum_{e \geq 1} \varphi_{D}^{(e)}\left(F_{*}^{e}\left(\mathfrak{a}^{\left\lceil\lambda\left(p^{e}-1\right)\right\rceil} c \cdot \mathcal{O}_{Y}\left(\left(1-p^{e}\right)\left(K_{Y}+D\right)\right)\right)\right)
$$

(see [ST, Proposition 6.8]). For example, if $u \in \mathfrak{a} \backslash\{0\}$ is such that $U=$ Spec $R_{u}$ is regular, and $\left.D\right|_{U}=0$, then one can take $c$ to be a power of $u$ (see [ST, Remark 6.6]).

Note that if $\mathfrak{a} \subseteq \mathfrak{b}$ and if $c \in R$ satisfy (12) for $\mathfrak{a}$, then it also satisfies (12) for $\mathfrak{b}$. An immediate consequence of the description (13) for the test ideal is the following monotonicity property. 
Proposition 3.10. If $(Y, D)$ is as above, and if $\mathfrak{a}, \mathfrak{b}$ are nonzero ideals on $Y$ with $\mathfrak{a} \subseteq \mathfrak{b}$, then $\tau\left(Y, D, \mathfrak{a}^{\lambda}\right) \subseteq \tau\left(Y, D, \mathfrak{b}^{\lambda}\right)$ for every $\lambda \in \mathbf{R}_{\geq 0}$.

The definition we gave for $\tau\left(Y, D, \mathfrak{a}^{\lambda}\right)$ applies when $D$ is an effective divisor. On the other hand, if $D^{\prime}$ is any effective Cartier divisor, then

$$
\tau\left(Y, D+D^{\prime}, \mathfrak{a}^{\lambda}\right)=\tau\left(Y, D, \mathfrak{a}^{\lambda}\right) \cdot \mathcal{O}_{Y}\left(-D^{\prime}\right)
$$

(see [ST, Lemma 6.11]). If $D$ is a not necessarily effective Weil divisor such that $K_{Y}+D$ is Cartier, then we define $\tau\left(Y, D, \mathfrak{a}^{\lambda}\right)$ as follows. Working locally, we can find a Cartier divisor $D^{\prime}$ such that $D+D^{\prime}$ is effective, and in this case $\tau\left(Y, D, \mathfrak{a}^{\lambda}\right)$ is the fractional ideal $\tau\left(Y, D+D^{\prime}, \mathfrak{a}^{\lambda}\right) \cdot \mathcal{O}_{Y}\left(D^{\prime}\right)$. It follows from (14) that the definition is independent of the choice of $D^{\prime}$.

If $Y$ is nonsingular, one can show that the above definition for the test ideal $\tau\left(Y, D, \mathfrak{a}^{\lambda}\right)$ coincides with the one in [BMS], which is the one we gave in Section 1. We refer to [BSTZ, Proposition 3.10] for a proof.

While we will not need the results on the jumping numbers for the test ideals, we mention them because of the analogy with the case of multiplier ideals. For the proofs, see [BMS] for the case when $Y$ is smooth and $D=0$, and see [BSTZ] for the general case. Given any $(Y, D, \mathfrak{a})$ as above, and any $\lambda \geq 0$, there is $\varepsilon>0$ such that $\tau\left(Y, D, \mathfrak{a}^{\lambda}\right)=\tau\left(Y, D, \mathfrak{a}^{\mu}\right)$ for every $\mu$ with $\lambda \leq \mu \leq \lambda+\varepsilon$. A positive $\lambda$ is an F-jumping number if $\tau\left(Y, D, \mathfrak{a}^{\lambda}\right) \neq$ $\tau\left(Y, D, \mathfrak{a}^{\mu}\right)$ for every $\mu<\lambda$. One can show that the set of $F$-jumping numbers is a discrete set of rational numbers. However, we emphasize that this result is much more subtle than the corresponding one for multiplier ideals.

The following proposition gives the analogue of Skoda's theorem for test ideals (see [BSTZ, Lemma 3.26]). For the smooth case, which is the only one that we will need in this paper, see [BMS, Proposition 2.25].

Proposition 3.11. Let $(Y, D, \mathfrak{a})$ be a triple as above, and let $m$ be a positive integer such that $\mathfrak{a}$ is locally generated by $m$ sections. For every $\lambda \geq m$ we have

$$
\tau\left(Y, D, \mathfrak{a}^{\lambda}\right)=\mathfrak{a} \cdot \tau\left(Y, D, \mathfrak{a}^{\lambda-1}\right) .
$$

We will make use in Section 6 of the following result of Schwede and Tucker [ST, Corollary 6.28] concerning the behavior of test ideals under finite morphisms. Let $\mu: Y^{\prime} \rightarrow Y$ be a finite surjective morphism of normal, irreducible varieties. Given the Weil divisor $D$ on $Y$ such that $K_{Y}+D$ is Cartier, then as in Proposition 3.2 we have a divisor $D_{Y^{\prime}}$ on $Y^{\prime}$ such that $K_{Y^{\prime}}+D_{Y^{\prime}}$ and $\mu^{*}\left(K_{Y}+D\right)$ are linearly equivalent. We also put $\mathfrak{a}^{\prime}=\mathfrak{a} \cdot \mathcal{O}_{Y^{\prime}}$. 
THEOREM 3.12. With the above notation, if $\mu$ is a separable morphism and if the trace map $\operatorname{Tr}: K\left(Y^{\prime}\right) \rightarrow K(Y)$ is surjective, then

$$
\tau\left(Y, D, \mathfrak{a}^{\lambda}\right)=\mu_{*} \tau\left(Y^{\prime}, D_{Y^{\prime}},\left(\mathfrak{a}^{\prime}\right)^{\lambda}\right) \cap K(Y) .
$$

Furthermore, if $D$ is effective, then

$$
\tau\left(Y, D, \mathfrak{a}^{\lambda}\right)=\mu_{*} \tau\left(Y^{\prime}, D_{Y^{\prime}},\left(\mathfrak{a}^{\prime}\right)^{\lambda}\right) \cap \mathcal{O}_{Y}
$$

One can compare this result with the corresponding result about multiplier ideals in Proposition 3.2. Note that the hypothesis in Theorem 3.12 is satisfied if $p=\operatorname{char}(L)$ does not divide $\left[K\left(Y^{\prime}\right): K(Y)\right]$.

\section{$\S 4$. The conjectural connection between multiplier ideals and test ideals}

The following is the main conjecture relating multiplier ideals and test ideals.

CONJECTURE 4.1. Let $Y$ be a normal, irreducible scheme over an algebraically closed field $k$ of characteristic zero. Suppose that $D$ is a Weil divisor on $Y$ such that $K_{Y}+D$ is Cartier, and suppose that $\mathfrak{a}$ is a nonzero ideal on $Y$. Given a model $Y_{A}$ of $Y$ over a ring $A \subset k$ of finite type over $\mathbf{Z}$, there is a dense set of closed points $S \subset \operatorname{Spec} A$ such that

$$
\tau\left(Y_{s}, D_{s}, \mathfrak{a}_{s}^{\lambda}\right)=\mathcal{J}\left(Y, D, \mathfrak{a}^{\lambda}\right)_{s} \quad \text { for all } \lambda \in \mathbf{R}_{\geq 0} \text { and all } s \in S
$$

Furthermore, if we have finitely many triples as above $\left(Y^{(i)}, D^{(i)}, \mathfrak{a}^{(i)}\right)$, and corresponding models over $\operatorname{Spec} A$, then there is a dense open subset of closed points in Spec $A$ such that (15) holds for each of these triples.

One can formulate variants of the above conjecture in more general settings. For example, $D$ may be assumed to be a $\mathbf{Q}$-divisor such that some multiple of $K_{Y}+D$ is Cartier, and one can replace the ideal $\mathfrak{a}$ by finitely many ideals $\mathfrak{a}_{1}, \ldots, \mathfrak{a}_{r}$. In the latter case one has to consider the corresponding mixed multiplier and test ideals. On the other hand, in our main result we will restrict ourselves to the case when $X$ is nonsingular. In particular, in this case $D$ is Cartier, and therefore (15) holds if and only if it holds when $D=0$. Therefore, in this case Conjecture 4.1 reduces to Conjecture 1.2. For examples related to the above conjecture in the case of an ambient nonsingular variety, see [Mus, Section 3] and [MTW, Section 4]. 
The inclusion $\subseteq$ in $(15)$ is due to Hara and Yoshida [HY]. In fact, this inclusion holds for an open subset of closed points in Spec $A$. It is a consequence of the more precise result below. We include a proof, since this is particularly easy with the alternative definition of test ideals that we are using.

Proposition 4.2. Let $Y$ be a normal irreducible scheme over a perfect field $L$ of positive characteristic $p$. Suppose that $D$ is a divisor on $Y$ such that $K_{Y}+D$ is Cartier, and suppose that $\mathfrak{a}$ is a nonzero ideal on $Y$. If $\pi: X \rightarrow Y$ is a proper birational morphism, with $X$ nonsingular, $\mathfrak{a} \cdot \mathcal{O}_{X}=$ $\mathcal{O}_{X}(-G)$ for a divisor $G$, and if $\operatorname{Supp}(G) \cup \operatorname{Supp}\left(D_{X}\right)$ has simple normal crossings, where the divisor $D_{X}$ on $X$ is defined as in Section 3.1, then

$$
\tau\left(Y, D, \mathfrak{a}^{\lambda}\right) \subseteq \pi_{*} \mathcal{O}_{X}\left(-D_{X}-\lfloor\lambda G\rfloor\right)
$$

for every $\lambda \in \mathbf{R}_{\geq 0}$.

Proof. After replacing $Y$ by each of the elements of a suitable open cover of $Y$, we may assume that there is a Cartier divisor $D^{\prime}$ on $Y$ such that $D+D^{\prime}$ is effective. Since it is enough to prove (16) with $D$ replaced by $D+D^{\prime}$, we may assume that $D$ is effective.

Let $J$ denote the right-hand side of (16). It follows from the minimality in the definition of the test ideal that in order to prove the inclusion in (16), it is enough to show that for every $e \geq 1$ we have the inclusion in (11). Let us fix such $e$. Since $X$ is nonsingular and $\operatorname{Supp}(G) \cup \operatorname{Supp}\left(D_{X}\right)$ has simple normal crossings, if $\mathfrak{b}=\mathcal{O}_{X}(-G)$, then $J^{\prime}:=\tau\left(X, D_{X}, \mathfrak{b}^{\lambda}\right)=\mathcal{O}_{X}\left(-D_{X}-\right.$ $\lfloor\lambda G\rfloor)$. Indeed, this is an easy consequence of (13) and of the formula in Example 3.7. (Note that in this case the $c$ in (13) can be taken to be a power of the defining equation of $\operatorname{Supp}\left(D_{X}\right) \cup \operatorname{Supp}(F)$.)

By definition, we have

$$
\varphi_{D_{X}}^{(e)}\left(F_{*}^{e}\left(\mathfrak{b}^{\ell} J^{\prime} \cdot \pi^{*}(\mathcal{L})\right)\right) \subseteq J^{\prime}
$$

where $\mathcal{L}=\mathcal{O}_{Y}\left(\left(1-p^{e}\right)\left(K_{Y}+D\right)\right)$ and $\ell=\left\lceil\lambda\left(p^{e}-1\right)\right\rceil$. We now use the commutativity of $(10)$. With the notation therein, we have $\rho\left(\mathfrak{a}^{\ell} J \cdot \mathcal{L}\right) \subseteq$ $\pi_{*}\left(\mathfrak{b}^{\ell} J^{\prime} \cdot \pi^{*}(\mathcal{L})\right)$. Therefore,

$$
\begin{aligned}
\varphi_{D}^{(e)}\left(F_{*}^{e}\left(\mathfrak{a}^{\ell} J \cdot \mathcal{L}\right)\right) & =\pi_{*}\left(\varphi_{D_{X}}^{(e)}\right)\left(F_{*}^{e}(\rho)\left(F_{*}^{e}\left(\mathfrak{a}^{\ell} J \cdot \mathcal{L}\right)\right)\right) \\
& \subseteq \pi_{*}\left(\varphi_{D_{X}}^{(e)}\right)\left(F_{*}^{e}\left(\rho\left(\mathfrak{a}^{\ell} J \cdot \mathcal{L}\right)\right)\right)
\end{aligned}
$$




$$
\begin{aligned}
& \subseteq \pi_{*}\left(\varphi_{D_{X}}^{(e)}\left(F_{*}^{e}\left(\mathfrak{b}^{\ell} J^{\prime} \cdot \pi^{*}(\mathcal{L})\right)\right)\right) \\
& \subseteq \pi_{*}\left(J^{\prime}\right)=J
\end{aligned}
$$

where the last inclusion follows by applying $\pi_{*}$ to (17). Therefore we have the inclusion in (11) for $J$, and this completes the proof of the proposition.

Note that in the setting of the conjecture, it is known that if we fix $\lambda$, then we get the equality in (15) for all closed points in an open subset of Spec $A$ (depending on $\lambda$ ). This was proved by Hara and Yoshida in [HY], relying on ideas that had been used also in $[\mathrm{Ha}]$ and $[\mathrm{MS}]^{*}$.

We end this section with the following proposition, which allows us to consider Conjecture 1.2 only in the case of principal ideals on nonsingular affine varieties.

Proposition 4.3. In order to prove Conjecture 1.2, it is enough to consider the case when $Y$ is an affine nonsingular variety and $\mathfrak{a}=(f)$ is a principal ideal (but allowing several such pairs).

Proof. Since every $Y$ admits a finite affine open cover $Y=\bigcup_{i} U_{i}$, and since proving the conjecture for $(Y, \mathfrak{a})$ is equivalent to proving it (simultaneously) for all $\left(U_{i},\left.\mathfrak{a}\right|_{U_{i}}\right)$, it follows that it is enough to consider the case when for all pairs we treat, the ambient scheme $Y$ is affine and nonsingular.

For such a pair $(Y, \mathfrak{a})$, let $h_{1}, \ldots, h_{m}$ be generators of $\mathfrak{a}$. It follows from Proposition 4.2 that we only need to guarantee the inclusion

$$
\mathcal{J}\left(Y, \mathfrak{a}^{\lambda}\right)_{s} \subseteq \tau\left(Y_{s}, \mathfrak{a}_{s}^{\lambda}\right) .
$$

Furthermore, in light of Propositions 3.6 and 3.11, it is enough to consider only the case $\lambda<m$.

Let $g_{1}, \ldots, g_{m}$ be general linear combinations of the $h_{i}$ with coefficients in $k$, and let $g=\prod_{i=1}^{m} g_{i}$, so that by Proposition 3.5 we have $\mathcal{J}\left(Y, \mathfrak{a}^{\lambda}\right)=$ $\mathcal{J}\left(Y, g^{\lambda / m}\right)$ for all $\lambda<m$. As we have seen in Section 3.2, in the case of multiplier ideals of bounded exponents we have only to consider finitely many such exponents (the candidate jumping numbers); hence, we may assume after taking a model over $A$ that for every closed point $s \in \operatorname{Spec} A$ we have

$$
\mathcal{J}\left(Y, \mathfrak{a}^{\lambda}\right)_{s}=\mathcal{J}\left(Y, g^{\lambda / m}\right)_{s}
$$

for all $\lambda<m$.

${ }^{*}$ The result in $[\mathrm{HY}]$ treats only the case of a local ring, since one uses the tight closure approach to test ideals. However, one can modify the proof therein to give the assertion in our setting. 
Suppose now that we can find a dense set $S$ of closed points in $\operatorname{Spec} A$ such that

$$
\mathcal{J}\left(Y, g^{\lambda / m}\right)_{s} \subseteq \tau\left(Y_{s}, g_{s}^{\lambda / m}\right)
$$

for every $s \in S$ and every $\lambda<m$. Since $g \in \mathfrak{a}^{m}$, we have by Propositions 3.8 and 3.10

$$
\tau\left(Y_{s}, g_{s}^{\lambda / m}\right) \subseteq \tau\left(Y_{s},\left(\mathfrak{a}_{s}^{m}\right)^{\lambda / m}\right) \subseteq \tau\left(Y_{s}, \mathfrak{a}_{s}^{\lambda}\right)
$$

for every $s \in S$. Putting together (19), (20), and (21), we get (18), which completes the proof of the proposition.

\section{$\S 5$. A conjecture regarding the Frobenius action on the cohomology of the structure sheaf}

In this section we discuss our conjecture about Frobenius actions and deduce some consequences. Let $k$ be an algebraically closed field of characteristic zero. We will freely use the notation and notions introduced in Section 2.1 and Section 2.2. Recall Conjecture 1.1: Suppose that $X$ is a connected, nonsingular $n$-dimensional projective algebraic variety over $k$, and that $X_{A}$ is a model of $X$ over the finitely generated $\mathbf{Z}$-subalgebra $A$ of $k$. Conjecture 1.1 asserts that there is a dense set of closed points $S \subset \operatorname{Spec} A$ such that the Frobenius action $F: H^{n}\left(X_{s}, \mathcal{O}_{X_{s}}\right) \rightarrow H^{n}\left(X_{s}, \mathcal{O}_{X_{s}}\right)$ is semisimple for every $s \in S$.

REMARK 5.1. In fact, one expects that the analogous assertion would be true for the Frobenius action on each of the cohomology vector spaces $H^{i}\left(X_{s}, \mathcal{O}_{X_{s}}\right)$. Moreover, it is expected that there is a dense set of closed points $s \in \operatorname{Spec} A$ such that each $X_{s}$ is ordinary in the sense of Bloch and Kato $[\mathrm{BK}]$ (see also [CL, exposé III] for a nice introduction to ordinary varieties). As follows from [BK, Proposition 7.3], if $X_{s}$ is ordinary, then the action of Frobenius on the Witt vector cohomology $H^{i}\left(X_{s}, W \mathcal{O}_{X_{s}}\right)$ is bijective. Note that we have an exact sequence of sheaves of abelian groups

$$
0 \rightarrow W \mathcal{O}_{X_{s}} \stackrel{V}{\rightarrow} W \mathcal{O}_{X_{s}} \rightarrow \mathcal{O}_{X_{s}} \rightarrow 0
$$

that is compatible with the action of Frobenius, where $V$ is the Verschiebung operator. From the long exact sequence

$$
H^{i}\left(X_{s}, W \mathcal{O}_{X_{s}}\right) \rightarrow H^{i}\left(X_{s}, W \mathcal{O}_{X_{s}}\right) \rightarrow H^{i}\left(X_{s}, \mathcal{O}_{X_{s}}\right)
$$




$$
\rightarrow H^{i+1}\left(X_{s}, W \mathcal{O}_{X_{s}}\right) \rightarrow H^{i+1}\left(X_{s}, W \mathcal{O}_{X_{s}}\right)
$$

that is compatible with the action of Frobenius, and the 5-lemma, it follows that Frobenius acts bijectively on $H^{i}\left(X_{s}, \mathcal{O}_{X_{s}}\right)$.

However, our hope is that proving that the Frobenius action on $H^{n}\left(X_{s}, \mathcal{O}_{X_{s}}\right)$ is semisimple will be easier than showing that $X_{s}$ is ordinary.

REMARK 5.2. If Conjecture 1.1 holds, then given finitely many varieties $X^{(1)}, \ldots, X^{(r)}$ as above, with $\operatorname{dim}\left(X^{(i)}\right)=d_{i}$, we may consider models $X_{A}^{(1)}, \ldots, X_{A}^{(r)}$ over $A$. In this case, there is a dense set of closed points $S \subset$ Spec $A$ such that the action of $F$ on each cohomology group $H^{d_{i}}\left(X_{s}^{(i)}, \mathcal{O}_{X_{s}^{(i)}}\right)$, with $s \in S$, is semisimple. Indeed, it is enough to apply the conjecture for $X=X^{(1)} \times \cdots \times X^{(r)}$, using Remark 2.3 and the fact that by Künneth's formula we have

$$
H^{d}\left(X_{s}, \mathcal{O}_{X_{s}}\right)=\bigotimes_{i=1}^{r} H^{d_{i}}\left(X_{s}^{(i)}, \mathcal{O}_{X_{s}^{(i)}}\right)
$$

where $d=\operatorname{dim}(X)=\sum_{i=1}^{r} d_{i}$.

Proposition 5.3. In order to prove Conjecture 1.1 for every algebraically closed field $k$ of characteristic zero, it is enough to prove it for the field of algebraic numbers $k=\overline{\mathbf{Q}}$.

Proof. Suppose that $X$ is defined over $k$, and let $X_{A}$ be a model over $A$, where $A \subset k$ is a $\mathbf{Z}$-algebra of finite type. As pointed out in Remark 2.5, it is enough to show that there is a closed point $s \in \operatorname{Spec} A$ such that the Frobenius action on $H^{n}\left(X_{s}, \mathcal{O}_{X_{s}}\right)$ is semisimple.

The $\mathbf{Q}$-algebra $A_{\mathbf{Q}}:=A \otimes_{\mathbf{Z}} \mathbf{Q}$ is finitely generated; hence, if $\mathfrak{m}$ is a prime ideal of $A$ such that $\mathfrak{m} A_{\mathrm{Q}}$ is a maximal ideal of $A_{\mathrm{Q}}$, then $K=A_{\mathrm{Q}} / \mathfrak{m} A_{\mathrm{Q}}$ is a finite extension of $\mathbf{Q}$. If $O_{K}$ is the ring of integers in $K$, then using the finite generation of $A$ over $\mathbf{Z}$ we see that there is a nonzero $h \in O_{K}$ such that the surjective morphism $A_{\mathbf{Q}} \rightarrow K$ induces a morphism $A \rightarrow B=\left(O_{K}\right)_{h}$.

Let $X_{B}=X_{A} \times_{\operatorname{Spec} A} \operatorname{Spec} B$, and let $X_{\overline{\mathbf{Q}}}=X_{A} \times_{\operatorname{Spec} A} \operatorname{Spec} \overline{\mathbf{Q}}$, where the morphism $A \rightarrow \overline{\mathbf{Q}}$ is given by the composition $A \rightarrow K \hookrightarrow \overline{\mathbf{Q}}$. Since we may assume that $X_{A}$ is smooth and projective over $\operatorname{Spec} A$, with geometrically connected generic fiber, it follows that $X_{\overline{\mathbf{Q}}}$ is connected, smooth, and projective over $\overline{\mathbf{Q}}$, and clearly $X_{B}$ is a model of $X_{\overline{\mathbf{Q}}}$ over $\operatorname{Spec} B$. If we know Conjecture 1.1 over $\overline{\mathbf{Q}}$, then it follows that there is a closed point 
$t \in \operatorname{Spec} B$ such that the Frobenius action on $H^{n}\left(\left(X_{\overline{\mathbf{Q}}}\right)_{t}, \mathcal{O}_{\left(X_{\overline{\mathbf{Q}}}\right)_{t}}\right)$ is semisimple. If $s \in \operatorname{Spec} A$ is the image of $t$, then we have a finite field extension $k(s) \hookrightarrow k(t)$, and $\left(X_{\overline{\mathbf{Q}}}\right)_{t}=X_{s} \times_{\operatorname{Spec} k(s)} \operatorname{Spec} k(t)$. Since $H^{n}\left(\left(X_{\overline{\mathbf{Q}}}\right)_{t}, \mathcal{O}_{\left(X_{\overline{\mathbf{Q}}}\right)}\right) \simeq$ $H^{n}\left(X_{s}, \mathcal{O}_{X_{s}}\right) \bigotimes_{k(s)} k(t)$, we conclude that the Frobenius action on $H^{n}\left(X_{s}, \mathcal{O}_{X_{s}}\right)$ is semisimple.

EXAmPLE 5.4. If $X$ is a $g$-dimensional abelian variety, then we may assume that $X_{s}$ is an abelian variety over $k(s)$ for every closed point $s \in$ Spec $A$. In this case $h^{1}\left(X_{s}, \mathcal{O}_{X_{s}}\right)=g$, and the action of Frobenius on $H^{g}\left(X_{s}, \mathcal{O}_{X_{s}}\right) \simeq \wedge^{g} H^{1}\left(X_{s}, \mathcal{O}_{X_{s}}\right)$ is semisimple if and only if the action of Frobenius on $H^{1}\left(X_{s}, \mathcal{O}_{X_{s}}\right)$ is semisimple. This is the case if and only if $X_{s}$ is ordinary in the usual sense, that is, if $X_{s} \times_{\operatorname{Spec} k(s)} \operatorname{Spec} \overline{k(s)}$ has $p^{g}$ $p$-torsion points, where $p=\operatorname{char}(k(s))$.

By Proposition 5.3, in order to check Conjecture 1.1 in this case we may assume that $X$ is defined over $\overline{\mathbf{Q}}$. The conjecture is then known if $g \leq 2$, but it is open in general. The case of elliptic curves is classical, while the case $g=2$ is due to Ogus [Og, Proposition 2.7] (see also [CL, théorème 6.3] for a proof of this result).

EXAMPLE 5.5. If $X$ is a smooth projective curve of genus $g$, then the action of Frobenius on $H^{1}\left(X_{s}, \mathcal{O}_{X_{s}}\right)$ is semisimple if and only if the Jacobian of $X_{s}$ is ordinary in the usual sense. As pointed out in the previous example, Conjecture 1.1 is known in this case for $g \leq 2$, but it is open even in this case for $g \geq 3$.

In what follows we will assume Conjecture 1.1 (for all smooth, connected projective varieties) and then deduce several stronger versions, working in the relative setting, and in the presence of a simple normal crossings divisor. We start by considering a pair $(X, E)$, where $X$ is a connected, nonsingular $n$-dimensional projective variety over $k$, and $E=E_{1}+\cdots+E_{r}$ is a reduced simple normal crossings divisor on $X$. Let $X_{A}$ be a model of $X$ over $\operatorname{Spec} A$. We may assume that $X_{A}$ is smooth over $A$ and that we have irreducible divisors $\left(E_{i}\right)_{A}$ on $X_{A}$ giving models for the $E_{i}$, such that every intersection $\left(E_{i_{1}}\right)_{A} \cap \cdots \cap\left(E_{i_{m}}\right)_{A}$ is smooth over $A$. In particular, if we put $E_{A}=\sum_{i=1}^{r}\left(E_{i}\right)_{A}$, then for every closed point $s \in \operatorname{Spec} A$, the divisor $E_{s}$ on $X_{s}$ has simple normal crossings.

LEMMA 5.6. With the above notation, if Conjecture 1.1 holds, then there is a dense set of closed points $S \subset \operatorname{Spec} A$ such that the Frobenius action $F: H^{n-1}\left(E_{s}, \mathcal{O}_{E_{s}}\right) \rightarrow H^{n-1}\left(E_{s}, \mathcal{O}_{E_{s}}\right)$ is semisimple for all $s \in S$. 
Proof. Let us fix a closed point $s \in \operatorname{Spec} A$. For every subset $J \subseteq\{1, \ldots, r\}$ we put $\left(E_{J}\right)_{s}=\bigcap_{i \in J}\left(E_{i}\right)_{s}$. (Of course, these sets will be empty for some $J$. .) Note that we have an acyclic complex

$$
C^{\bullet}: 0 \rightarrow C^{0} \stackrel{d^{0}}{\rightarrow} C^{1} \stackrel{d^{1}}{\rightarrow} \cdots \stackrel{d^{n-1}}{\rightarrow} C^{n} \rightarrow 0,
$$

where $C^{0}=\left(\mathcal{O}_{E}\right)_{s}$, and for all $p>0$ we have $C^{p}=\bigoplus_{|J|=p} \mathcal{O}_{\left(E_{J}\right)_{s}}$. Furthermore, we have a morphism of complexes $C^{\bullet} \rightarrow F_{*} C^{\bullet}$. If we put $Z^{i}=\operatorname{Ker}\left(d^{i}\right)$ for $1 \leq i \leq n-1$ and $Z^{n}=C^{n}$, then we have exact sequences

$$
H^{n-p-1}\left(X_{s}, Z^{p+1}\right) \rightarrow H^{n-p}\left(X_{s}, Z^{p}\right) \rightarrow H^{n-p}\left(X_{s}, C^{p}\right)
$$

compatible with the action of Frobenius. Applying Conjecture 1.1 to all connected components of all the intersections $E_{i_{1}} \cap \cdots \cap E_{i_{m}}$ simultaneously (see Remark 5.2), we see that we have a dense set of closed points $S \subset \operatorname{Spec} A$ such that the Frobenius action on each $H^{n-p}\left(X_{s}, C^{p}\right)$ is semisimple for $p \geq 1$ and $s \in S$. Using Lemma 2.4 and the exact sequences (22), we see by descending induction on $p \leq n$ that for every $s \in S$, the Frobenius action on each $H^{n-p}\left(X_{s}, Z^{p}\right)$ is semisimple. By taking $p=1$, we get the assertion in the lemma.

Corollary 5.7. With the notation in the lemma, and still assuming Conjecture 1.1, there is a dense set of closed points $S \subset \operatorname{Spec} A$ such that the Frobenius action

$$
F: H^{n}\left(X_{s}, \mathcal{O}\left(-E_{s}\right)\right) \rightarrow H^{n}\left(X_{s}, \mathcal{O}\left(-E_{s}\right)\right)
$$

is semisimple for every $s \in S$.

Proof. Consider the exact sequence

$$
T^{\bullet}: 0 \rightarrow \mathcal{O}_{X_{s}}\left(-E_{s}\right) \rightarrow \mathcal{O}_{X_{s}} \rightarrow \mathcal{O}_{E_{s}} \rightarrow 0 .
$$

Note that we have a morphism of exact sequences $T^{\bullet} \rightarrow F_{*} T^{\bullet}$. Applying Lemma 2.4 to the exact sequence

$$
H^{n-1}\left(E_{s}, \mathcal{O}_{E_{s}}\right) \rightarrow H^{n}\left(X_{s}, \mathcal{O}_{X_{s}}\left(-E_{s}\right)\right) \rightarrow H^{n}\left(X_{s}, \mathcal{O}_{X_{s}}\right),
$$

as well as Conjecture 1.1 to $H^{n}\left(X_{s}, \mathcal{O}_{X_{s}}\right)$ and Lemma 5.6 to $H^{n-1}\left(E_{s}, \mathcal{O}_{E_{s}}\right)$ (note that we can apply these simultaneously by Remark 5.2), it follows that the Frobenius action on $H^{n}\left(X_{s}, \mathcal{O}\left(-E_{s}\right)\right)$ is semisimple for all $s$ in a suitable dense set of closed points $S \subset \operatorname{Spec} A$. 
Still keeping the above notation, let $s \in \operatorname{Spec} A$ be a closed point. Recall that we have a canonical surjective $\mathcal{O}_{X_{s}}$-linear map $t_{s}:=t_{X_{s}, E_{s}}$ : $F_{*}\left(\omega_{X_{s}}\left(E_{s}\right)\right) \rightarrow \omega_{X_{s}}\left(E_{s}\right)$ induced by the Cartier isomorphism. For every $e \geq 1$ we also consider the composition $t_{s}^{e}: F_{*}^{e}\left(\omega_{X_{s}}\left(E_{s}\right)\right) \rightarrow \omega_{X_{s}}\left(E_{s}\right)$.

COROllary 5.8. With the notation in Lemma 5.6, and assuming that Conjecture 1.1 holds, there is a dense set of closed points $S \subset \operatorname{Spec} A$ such that the map induced by $t_{s}^{e}$

$$
H^{0}\left(X_{s}, F_{*}^{e}\left(\omega_{X_{s}}\left(E_{s}\right)\right)\right) \rightarrow H^{0}\left(X_{s}, \omega_{X_{s}}\left(E_{s}\right)\right)
$$

is surjective for all $e \geq 1$ and all $s \in S$.

Proof. It is enough to show that every closed point $s \in \operatorname{Spec} A$ that satisfies Corollary 5.7 also satisfies our conclusion. As abelian groups, we have $H^{0}\left(X_{s}, F_{*}^{e}\left(\omega_{X_{s}}\left(E_{s}\right)\right)\right)=H^{0}\left(X_{s}, \omega_{X_{s}}\left(E_{s}\right)\right)$, and the map induced by $t_{s}^{e}$ is just the $e$ th iterate of the map induced by $t_{s}$. Therefore, it is enough to prove the assertion in the case $e=1$. On the other hand, this case follows if we show the surjectivity when $e$ is such that the cardinality of the residue field $k(s)$ is $p^{e}$. Note that in this case the map $t_{s}^{e}: H^{0}\left(X_{s}, \omega_{X_{s}}\left(E_{s}\right)\right) \rightarrow H^{0}\left(X_{s}, \omega_{X_{s}}\left(E_{s}\right)\right)$ is $k(s)$-linear. Its Serre dual is the map

$$
F^{e}: H^{n}\left(X_{s}, \mathcal{O}_{X_{s}}\left(-E_{s}\right)\right) \rightarrow H^{0}\left(X_{s}, \mathcal{O}_{X_{s}}\left(-E_{s}\right)\right)
$$

where $F$ denotes the Frobenius action on $H^{n}\left(X_{s}, \mathcal{O}_{X_{s}}\left(-E_{s}\right)\right)$. By assumption, $F$ is semisimple, hence bijective, which implies the assertion in the lemma.

REMARK 5.9. It follows from the proofs of Lemma 5.6 and of Corollaries 5.7 and 5.8 that in order to get the assertions in these two corollaries we need to apply Conjecture 1.1 to finitely many smooth projective varieties. It follows from Remark 5.2 that if we have finitely many pairs $\left(X^{(1)}, E^{(1)}\right), \ldots,\left(X^{(m)}, E^{(m)}\right)$ as in Corollaries 5.7 and 5.8 , then we can find a dense set of closed points $s \in \operatorname{Spec} A$ such that the conclusion in each of these two corollaries holds for all these pairs. In particular, in Corollary 5.8 we do not need to assume that $X$ is connected.

We now turn to the relative setting and state the main result of this section.

Theorem 5.10. Suppose that Conjecture 1.1 holds. Let $\pi: X \rightarrow T$ be a projective morphism of schemes over $k$, with $X$ nonsingular, and let 
$E=E_{1}+\cdots+E_{r}$ be a reduced simple normal crossings divisor on $X$. If $\pi_{A}: X_{A} \rightarrow T_{A}$ and $E_{A}$ are models over $A$ for $\pi$ and $E$, respectively, then there is a dense set of closed points $S \subset \operatorname{Spec} A$ such that for every $e \geq 1$ and every $s \in S$, the induced morphism

$$
\left(\pi_{s}\right)_{*}\left(F_{*}^{e}\left(\omega_{X_{s}}\left(E_{s}\right)\right)\right) \rightarrow\left(\pi_{s}\right)_{*}\left(\omega_{X_{s}}\left(E_{s}\right)\right)
$$

is surjective.

Proof. Suppose first that $T$ is affine. Since $\pi$ is projective, we have a closed immersion $X \hookrightarrow \mathbf{P}^{N} \times T$, for some $N \geq 1$. Let us fix an open immersion $T \hookrightarrow T^{\prime}$, where $T^{\prime}$ is projective. Let $\bar{X}$ be the closure of $X$ in $\mathbf{P}^{N} \times T^{\prime}$ (with the reduced scheme structure), and let $\bar{\pi}: \bar{X} \rightarrow T^{\prime}$ be the induced morphism. Since $\bar{X} \cap\left(\mathbf{P}^{N} \times T\right)=X$, it follows that $\bar{\pi}^{-1}(T)=X$.

By hypothesis, $X$ is nonsingular and $E$ has simple normal crossings; hence, by the standard results on resolution of singularities in characteristic zero, there is a projective morphism $\varphi: X^{\prime} \rightarrow \bar{X}$ that is an isomorphism over $X$, with $X^{\prime}$ nonsingular, and a reduced simple normal crossings divisor $E^{\prime}$ on $X^{\prime}$ such that $\left.E^{\prime}\right|_{\varphi^{-1}(X)}=\varphi^{-1}(E)$. If $\pi^{\prime}=\bar{\pi} \circ \varphi$, then $X$ is isomorphic to $\left(\pi^{\prime}\right)^{-1}(T)$, and it is clear that if the assertion in Theorem 5.10 holds for $\pi^{\prime}$ and $E^{\prime}$, then it also holds for $\pi$ and $E$. Therefore, we may assume that $X$ and $T$ are projective.

We now choose a very ample line bundle $\mathcal{L}$ on $T$ such that $\pi_{*}\left(\omega_{X}(E)\right) \otimes \mathcal{L}$ is globally generated. After possibly replacing $A$ by some localization $A_{a}$, we may assume that for every closed point $s \in \operatorname{Spec} A$ we have $\left(\omega_{X}\right)_{s}=\omega_{X_{s}}$ and $\pi_{*}\left(\omega_{X}(E)\right)_{s}=\left(\pi_{s}\right)_{*}\left(\omega_{X_{s}}\left(E_{s}\right)\right)$. In particular, $\left(\pi_{s}\right)_{*}\left(\omega_{X_{s}}\left(E_{s}\right)\right) \otimes \mathcal{L}_{s}$ is globally generated.

Since $\mathcal{L}$ is very ample, the linear system $|\mathcal{L}|$ and its pullback to $X$ are globally generated. It follows from Bertini's theorem (recall that $\operatorname{char}(k)=$ $0)$ that if $D^{\prime} \in|\mathcal{L}|$ is a general element, then $E^{\prime}=\pi^{*}\left(D^{\prime}\right)$ has the property that $E+E^{\prime}$ is a reduced simple normal crossings divisor. Of course, it is enough to ensure that (23) is surjective after tensoring with $\mathcal{L}_{s}$, and since $\left(\pi_{s}\right)_{*}\left(\omega_{X_{s}}\left(E_{s}\right)\right) \otimes \mathcal{L}_{s}$ is globally generated, it is enough to show that the map

$$
H^{0}\left(T_{s},\left(\pi_{s}\right)_{*}\left(F_{*}^{e}\left(\omega_{X_{s}}\left(E_{s}\right)\right)\right) \otimes \mathcal{L}_{s}\right) \rightarrow H^{0}\left(T_{s},\left(\pi_{s}\right)_{*}\left(\omega_{X_{s}}\left(E_{s}\right)\right) \otimes \mathcal{L}_{s}\right)
$$

is surjective. By the projection formula, (24) gets identified with the map

$$
H^{0}\left(X_{s}, F_{*}^{e}\left(\omega_{X_{s}}\left(E_{s}+p^{e} E_{s}^{\prime}\right)\right)\right) \rightarrow H^{0}\left(X_{s}, \omega_{X_{s}}\left(E_{s}+E_{s}^{\prime}\right)\right) .
$$


Applying Corollary 5.8 to $X$ and $E+E^{\prime}$, we deduce that there is a dense set of closed points $S \subseteq \operatorname{Spec} A$ such that the composition

$$
\begin{aligned}
H^{0}\left(X_{s}, F_{*}^{e}\left(\omega_{X_{s}}\left(E_{s}+E_{s}^{\prime}\right)\right)\right) & \rightarrow H^{0}\left(X_{s}, F_{*}^{e}\left(\omega_{X_{s}}\left(E_{s}+p^{e} E_{s}^{\prime}\right)\right)\right) \\
& \rightarrow H^{0}\left(X_{s}, \omega_{X_{s}}\left(E_{s}+E_{s}^{\prime}\right)\right)
\end{aligned}
$$

is surjective for every $s \in S$. This clearly implies the surjectivity of (25) and completes the proof in the case when $T$ is affine.

Note that the proof in this special case relies on an application of Corollary 5.8 for one pair. In general, we consider a finite affine cover $T=\bigcup_{i} U_{i}$. Combining what we proved so far with Remark 5.9, we see that there is a dense set of closed points $S \subseteq \operatorname{Spec} A$ such that the assertion in the theorem holds for all morphisms $\pi^{-1}\left(U_{i}\right) \rightarrow U_{i}$ and for all $s \in S$. This implies the surjectivity of the map in (23) for every $s \in S$, which completes the proof of the theorem.

REMARK 5.11. It follows from the proof of Theorem 5.10, using also Remark 5.9, that given finitely many morphisms $\pi^{(i)}: X^{(i)} \rightarrow T^{(i)}$ and divisors $E^{(i)}$ on $X^{(i)}$ satisfying the hypothesis in the theorem, there is a dense set of closed points of Spec $A$ that satisfies the conclusion of the theorem with respect to each of the morphisms $\pi^{(i)}$.

\section{$\S 6$. The connection between the two conjectures}

We can now prove our main result, stated in Section 1.

Proof of Theorem 1.3. Let us assume that Conjecture 1.1 holds. Actually, we will use its consequence in Theorem 5.10. It follows from Proposition 4.3 that in order to show that Conjecture 1.2 holds, it is enough to consider the following setup. Suppose that $Y$ is a nonsingular, irreducible affine variety over an algebraically closed field $k$ of characteristic zero. Let $\mathfrak{a}=(f)$ be a nonzero principal ideal on $Y$. We need to show that given a model of $(Y, \mathfrak{a})$ over $A$, where $A$ is a subalgebra of $k$ of finite type over $\mathbf{Z}$, there is a dense set of closed points $S \subset \operatorname{Spec} A$ such that

$$
\tau\left(Y_{s}, f_{s}^{\lambda}\right)=\mathcal{J}\left(Y, f^{\lambda}\right)_{s}
$$

for all $s \in S$ and all $\lambda \in \mathbf{R}_{\geq 0}$. Furthermore, given finitely many such pairs $(Y, \mathfrak{a})$, we need to be able to do this simultaneously for all the pairs.

After covering $Y$ by suitable affine open subsets, we may assume that $f: Y \rightarrow \mathbf{A}^{1}$ is smooth over $\mathbf{A}^{1} \backslash\{0\}$. Indeed, there is an open neighborhood 
$U$ of $V(f)$ such that $f$ is smooth on $U \backslash V(f)$, while on $Y \backslash V(f)$ we may replace $f$ by 1 .

Therefore, we can apply the semistable reduction theorem of [KKMS] for $f$ to get a positive integer $d \geq 1$ with the following property. If $\beta: \mathbf{A}^{1} \rightarrow$ $\mathbf{A}^{1}$ is given by $\beta(t)=t^{d}$, and if we consider the Cartesian diagram

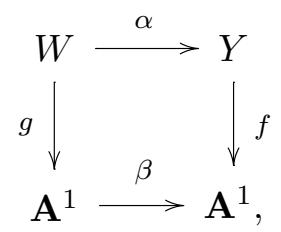

then there is a projective morphism $\psi: Z \rightarrow W$ that satisfies

(i) $\psi$ is an isomorphism over $\mathbf{A}^{1} \backslash\{0\}$ (in particular, $\psi$ is birational),

(ii) $Z$ is nonsingular,

(iii) $\psi^{*}(g)$ defines a reduced simple normal crossings divisor on $Z$.

Let $W_{0}$ be an irreducible component of $W$ that maps surjectively onto $Y$, and let $X$ be the corresponding irreducible component of $Z$ that surjects onto $W_{0}$. If $Y^{\prime}$ is the normalization of $W_{0}$, then we have induced morphisms

$$
X \stackrel{\pi}{\rightarrow} Y^{\prime} \stackrel{\varphi}{\rightarrow} Y
$$

We denote by $h$ the pullback of $g$ to $Y^{\prime}$. By construction, $\varphi$ is finite and surjective, étale over $Y \backslash V(f)$. In particular, the singular locus of $Y^{\prime}$ is contained in $V(h)$.

Let $D^{\prime}=-K_{Y^{\prime} / Y}$ be the divisor defined as in Proposition 3.2. Note that $D^{\prime}$ is supported on $V(h)$. It follows from Proposition 3.2 (see also Remark 3.3) that for every $\lambda \in \mathbf{R}_{\geq 0}$ we have

$$
\mathcal{J}\left(Y, f^{\lambda}\right)=\varphi_{*} \mathcal{J}\left(Y^{\prime}, D^{\prime}, h^{m \lambda}\right) \cap \mathcal{O}_{Y}
$$

We define the divisor $D_{X}^{\prime}$ as in Section 3.1, such that, in particular, $K_{X}+$ $D_{X}^{\prime}$ and $\pi^{*}\left(K_{Y^{\prime}}+D^{\prime}\right)$ are linearly equivalent. Let $E$ be the reduced simple normal crossings divisor defined on $X$ by $\pi^{*}(h)$. Note that $D_{X}^{\prime}$ is supported on $E$, which has simple normal crossings; hence, it follows from Proposition 3.1 and the definition of multiplier ideals that for every $\lambda \in \mathbf{R}_{\geq 0}$ we have

$$
\mathcal{J}\left(Y^{\prime}, D^{\prime}, h^{m \lambda}\right)=\pi_{*} \mathcal{O}_{X}\left(-D_{X}^{\prime}-\lfloor m \lambda E\rfloor\right) .
$$

We choose a model over a finitely generated $\mathbf{Z}$-algebra $A$, contained in $k$, for all the above varieties and morphisms. We may assume that the above 
properties extend to all fibers over $k(s)$, for $s \in \operatorname{Spec} A$ a closed point. Furthermore, after replacing $A$ by some localization $A_{a}$, we may assume that for every closed point $s \in \operatorname{Spec} A$ the characteristic of $k(s)$ does not divide $\left[K\left(Y^{\prime}\right): K(Y)\right]$. In this case Theorem 3.12 applies to give

$$
\tau\left(Y_{s}, f_{s}^{\lambda}\right)=\left(\varphi_{s}\right)_{*} \tau\left(Y_{s}^{\prime}, D_{s}^{\prime}, h_{s}^{m \lambda}\right) \cap \mathcal{O}_{Y_{s}}
$$

for every closed point $s \in \operatorname{Spec} A$ and every $\lambda \in \mathbf{R}_{\geq 0}$. On the other hand, we may assume that (28) induces

$$
\mathcal{J}\left(Y, f^{\lambda}\right)_{s}=\left(\varphi_{s}\right)_{*} \mathcal{J}\left(Y^{\prime}, D^{\prime}, h^{m \lambda}\right)_{s} \cap \mathcal{O}_{Y_{s}}
$$

and that (29) induces

$$
\mathcal{J}\left(Y^{\prime}, D^{\prime}, h^{m \lambda}\right)_{s}=\left(\pi_{s}\right)_{*} \mathcal{O}_{X_{s}}\left(-D_{X_{s}}^{\prime}-\left\lfloor m \lambda E_{s}\right\rfloor\right)
$$

for every $s \in \operatorname{Spec} A$ and every $\lambda \in \mathbf{R}_{\geq 0}$. Therefore, in order to guarantee $\tau\left(Y_{s}, f_{s}^{\lambda}\right)=\mathcal{J}\left(Y, f^{\lambda}\right)_{s}$ for all $\lambda$, it is enough to ensure that

$$
\tau\left(Y_{s}^{\prime}, D_{s}^{\prime}, h_{s}^{m \lambda}\right)=\left(\pi_{s}\right)_{*} \mathcal{O}_{X_{s}}\left(-D_{X_{s}}^{\prime}-\left\lfloor m \lambda E_{s}\right\rfloor\right)
$$

for all $\lambda \in \mathbf{R}_{\geq 0}$.

We now apply Theorem 5.10 to the morphism $\pi: X \rightarrow Y^{\prime}$ and to the reduced simple normal crossings divisor $E$. It follows that there is a dense set of closed points $S \subset \operatorname{Spec} A$ such that

$$
\left(\pi_{s}\right)_{*}\left(F_{*}^{e}\left(\omega_{X_{s}}\left(E_{s}\right)\right)\right) \rightarrow\left(\pi_{s}\right)_{*}\left(\omega_{X_{s}}\left(E_{s}\right)\right)
$$

is surjective for every $s \in S$ and every $e \geq 1$. The equality (33) now follows applying Lemma 6.1 below to the morphism $\pi_{s}: X_{s} \rightarrow Y_{s}^{\prime}$, the divisor $D_{s}^{\prime}$, and $h_{s} \in \Gamma\left(Y_{s}^{\prime}, \mathcal{O}_{Y_{s}^{\prime}}\right)$.

Lemma 6.1. Let $\pi: X \rightarrow T$ be a birational morphism of schemes of finite type over a perfect field of characteristic $p>0$, with $T$ normal and irreducible and $h \in \Gamma\left(T, \mathcal{O}_{T}\right)$ nonzero. If $D$ is a divisor on $T$ supported on $V(h)$ such that $K_{T}+D$ is Cartier, and if the following hold:

(i) $X$ is nonsingular;

(ii) $E:=\pi^{*}(\operatorname{div}(h))$ is a reduced divisor, with simple normal crossings;

(iii) $\pi$ is proper, and an isomorphism over $T \backslash V(h)$;

(iv) the map $\pi_{*}\left(F_{*}^{e}\left(\omega_{X}(E)\right)\right) \rightarrow \pi_{*}\left(\omega_{X}(E)\right)$ is surjective for every $e \geq 1$, 
then $\tau\left(T, D, h^{\lambda}\right)=\pi_{*} \mathcal{O}_{X}\left(-D_{X}-\lfloor\lambda E\rfloor\right)$ for every $\lambda \in \mathbf{R}_{\geq 0}$, where $D_{X}$ is defined as in Section 3.1.

Proof. Note that by (iii), the divisor $D_{X}$ is supported on $E$; hence, $D_{X}, E$ have simple normal crossings by (ii). Proposition 4.2 gives the inclusion $\subseteq$ in the statement; hence, we just need to show that

$$
\pi_{*} \mathcal{O}_{X}\left(-D_{X}-\lfloor\lambda E\rfloor\right) \subseteq \tau\left(T, D, h^{\lambda}\right)
$$

for every $\lambda \geq 0$.

After replacing $D$ by $D+m \cdot \operatorname{div}(h)$, with $m \gg 0$, we may assume that $D$ is effective. It follows from the projection formula and from Proposition 3.11 that it is enough to prove (34) for $\lambda<1$. Let us fix such $\lambda$. Note that in this case, the left-hand side of $(34)$ is equal to $\pi_{*} \mathcal{O}_{X}\left(-D_{X}\right)$. We write $D_{X}=\sum_{i=1}^{N} a_{i} E_{i}$.

After taking a finite affine open cover of $T$, we may assume that $T$ is affine. For the description of $\tau\left(T, D, h^{\lambda}\right)$ we use (13). Note that by (i) and (iii), the singular locus of $T$ is contained in $V(h)$. Since $D$ is also supported on $V(h)$, we see that if $\ell \gg 0$, then we may take $c=h^{\ell}$ in (13). We fix $\ell$ with this property such that, in addition, $\ell \geq a_{i}$ for all $i$. It follows that it is enough to show that if $e \gg 0$, then

$$
\pi_{*} \mathcal{O}_{X}\left(-D_{X}\right) \subseteq \varphi_{D}^{(e)}\left(F_{*}^{e}\left(h^{d_{e}} \cdot \mathcal{O}_{T}\left(\left(1-p^{e}\right)\left(K_{T}+D\right)\right)\right)\right)
$$

where $d_{e}=\left\lceil\lambda\left(p^{e}-1\right)\right\rceil+\ell$.

For the sake of a more compact notation, we denote $\mathcal{L}=\mathcal{O}_{T}\left(\left(1-p^{e}\right)\left(K_{T}+\right.\right.$ $D)$ ). We use the commutative diagram (10) to write the right-hand side of $(35)$ as

$$
\pi_{*}\left(\varphi_{D_{X}}^{(e)}\right)\left(F_{*}^{e}\left(\rho\left(h^{d_{e}} \cdot \mathcal{L}\right)\right)\right)
$$

for which we recall that $\rho: \mathcal{L} \rightarrow \pi_{*}\left(\pi^{*}(\mathcal{L})\right)$ denotes the canonical isomorphism. It is clear that $F_{*}^{e}\left(\rho\left(h^{d_{e}} \cdot \mathcal{L}\right)\right)=\pi_{*}\left(F_{*}^{e}\left(h^{d_{e}} \cdot \pi^{*}(\mathcal{L})\right)\right)$. For $e \gg 0, \varphi_{D_{X}}^{(e)}$ induces a surjection on $X$,

$$
u: F_{*}^{e}\left(h^{d_{e}} \cdot \pi^{*}(\mathcal{L})\right) \rightarrow \mathcal{O}_{X}\left(-D_{X}\right) .
$$

This follows from Example 3.7 and the fact that $\left\lfloor\left(d_{e}-a_{i}\right) / p^{e}\right\rfloor=0$ for $e \gg 0$.

Claim. $\pi_{*}(u): \pi_{*}\left(F_{*}^{e}\left(h^{d_{e}} \cdot \pi^{*}(\mathcal{L})\right)\right) \rightarrow \pi_{*} \mathcal{O}_{X}\left(-D_{X}\right)$ is surjective. 
If this holds, then the expression in (36) is equal to $\pi_{*} \mathcal{O}_{X}\left(-D_{X}\right)$, which gives the inclusion in (35).

Therefore, the proof of the lemma is complete if we show the claim. Note that the surjectivity of $\pi_{*}(u)$ is equivalent to the surjectivity of $\pi_{*}(u) \otimes$ $\mathcal{O}_{T}\left(K_{T}+D\right)$. Using the projection formula, this becomes equivalent to the surjectivity of

$$
\pi_{*}\left(F_{*}^{e}\left(\omega_{X}\left(D_{X}-d_{e} E\right)\right)\right) \rightarrow \pi_{*}\left(\omega_{X}\right) .
$$

For $e \gg 0$, the divisor $\left(D_{X}-d_{e} E\right)+\left(p^{e}-1\right) E$ is effective; hence, the surjectivity of the map in (38) follows from the surjectivity of

$$
w: \pi_{*}\left(F_{*}^{e}\left(\omega_{X}\left(-\left(p^{e}-1\right) E\right)\right)\right) \rightarrow \pi_{*}\left(\omega_{X}\right)
$$

This in turn is surjective if and only if $w \otimes \mathcal{O}_{T}(H)$ is surjective, but the latter map is identified via the projection formula with

$$
\pi_{*}\left(F_{*}^{e}\left(\omega_{X}(E)\right)\right) \rightarrow \pi_{*}\left(\omega_{X}(E)\right),
$$

which is surjective by the assumption in (iv). This completes the proof of the lemma.

Acknowledgments. We are indebted to Bhargav Bhatt, Hélène Esnault, and Johannes Nicaise for several inspiring discussions. We would also like to thank Karl Schwede and the anonymous referee, whose thoughtful comments helped improve the paper. Part of this work was done during the second author's visit to Ann Arbor. We are grateful to the University of Michigan and to the David and Lucile Packard Foundation for making this visit possible.

\section{REFERENCES}

[BMS] M. Blickle, M. Mustaţă, and K. E. Smith, Discreteness and rationality of F-thresholds, Michigan Math. J. 57 (2008), 463-483.

[BSTZ] M. Blickle, K. Schwede, S. Takagi, and W. Zhang, Discreteness and rationality of F-jumping numbers on singular varieties, Math. Ann. 347 (2010), 917-949.

[BK] S. Bloch and K. Kato, p-adic étale cohomology, Publ. Math. Inst. Hautes Études Sci. 63 (1986), 107-152.

[CL] A. Chambert-Loir, Cohomologie cristalline: un survol, Expo. Math. 16 (1998), 333382.

[DI] P. Deligne and L. Illusie, Relèvements modulo $p^{2}$ et décomposition du complexe de de Rham, Invent. Math. 89 (1987), 247-270.

[Eis] D. Eisenbud, Commutative Algebra. With a View toward Algebraic Geometry, Grad. Texts in Math. 150, Springer, New York, 1995. 
[Ha] N. Hara, A characterization of rational singularities in terms of injectivity of Frobenius maps, Amer. J. Math. 120 (1998), 981-996.

[HW] N. Hara and K. Watanabe, F-regular and F-pure rings vs. log terminal and log canonical singularities, J. Algebraic Geom. 11 (2002), 363-392.

[HY] N. Hara and K. Yoshida, A generalization of tight closure and multiplier ideals, Trans. Amer. Math. Soc. 355 (2003), 3143-3174.

[Hart] R. Hartshorne, Algebraic Geometry, Grad. Texts in Math. 52, Springer, New York, 1977.

[KKMS] G. Kempf, F. Knudsen, D. Mumford, and B. Saint-Donat, Toroidal Embeddings I, Lecture Notes in Math. 339, Springer, Berlin, 1973.

[Laz] R. Lazarsfeld, Positivity in Algebraic Geometry, II, Ergeb. Math. Grenzgeb. (3), Folge 49, Springer, Berlin, 2004.

[LLS] R. Lazarsfeld, K. Lee, and K. E. Smith, Syzygies of multiplier ideals on singular varieties, Michigan Math. J. 57 (2008), 511-521.

[MS] V. B. Mehta and V. Srinivas, A characterization of rational singularities, Asian J. Math. 1 (1997), 249-271.

[Mus] M. Mustaţă, Bernstein-Sato polynomials in positive characteristic, J. Algebra 321 (2009), 128-151.

[MTW] M. Mustaţă, S. Takagi, and K. Watanabe, "F-thresholds and Bernstein-Sato polynomials" in European Congress of Mathematics, Eur. Math. Soc., Zürich, 2005, $341-364$.

[Og] A. Ogus, "Hodge cycles and crystalline cohomology" in Hodge Cycles, Motives, and Shimura Varieties, Lecture Notes in Math. 900, Springer, Berlin, 357-414.

[Sch] K. Schwede, F-adjunction, Algebra Number Theory 3 (2009), 907-950.

[ST] K. Schwede and K. Tucker, On the behavior of test ideals under finite morphisms, preprint, arXiv:1003.4333v2 [math.AG]

[Smi] K. E. Smith, F-rational rings have rational singularities, Amer. J. Math. 119 (1997), 159-180.

\author{
Mircea Mustaţă \\ Department of Mathematics \\ University of Michigan \\ Ann Arbor, MI 48109 \\ $U S A$ \\ mmustata@umich.edu \\ Vasudevan Srinivas \\ School of Mathematics \\ Tata Institute of Fundamental Research \\ Colaba, Mumbai 400005 \\ India \\ srinivas@math.tifr.res.in
}

